\title{
Elev-Fortegnelse \\ fra \\ Christiansfelds Kostskoler 1775-1891.
}

Af Jens Holdt, Brede.

Nærværende Elev-Fortegnelse skal give et Billede af Herrnhuterbyen Chrisitansfelds Indflydelse udadtil paa Opdragelsens Omraade. Den spænder over godt eet Aarhundrede, nemlig fra Skolernes Oprettelse i 1775 til deres Overgang til offentlig, statskontrolleret Realskole i 1891, da samtidig Kostskoleordningen blev opgivet. Opført er alle Elever, der kom udefra til Christiansfeld, nemlig 1) Pensionærerne, der under Skoleopholdet - i Regelen de 3-4 Aar før Konfirmationen, i den første Tid dog helt fra 6 Aars Alderen - boede paa selve Skolen, deriblandt ogsaa Menighedens Diasporaarbejderog Missionærbørn, og 2) det med Aarene voksende Tal af saakaldte Dags k o l e-E l e ver, der boede hjemme eller var privat indlogerede paa Stedet og kun modtog Undervisningen paa Skolen, deriblandt ogsaa enkelte, der kun kom til Christiansfeld et halvt Aarstid for at forberedes til Konfirmationen (alle mærket med *). Ikke medtaget er Christiansfeld-Menighedens egne Børn, der paa Forhaand var henviste til Skolegang i Byen. Der anføres iøvrigt 1) Aaret for Elevens Indtrædelse, 2) Navn, 3) Fødselsaar, 4) Hjemsted, 5) Faderens Stilling. I Noter tilføjes om enkelte Elever nogle Oplysninger, der skønnes at have en vis personalhistorisk Interesse.

G e o g r a f i s k spænder Christiansfelds Skoleopland over et vældigt Landomraade, nemlig fra Moskva til New York og fra Grønland til Syd Afrika, betinget ved Brødremenighedens vidtspændende Missionsforetagender. Det egentlige Elev-Opland er dog de tre skandinaviske Hovedlande Danmark (med Hertugdømmerne), Norge og Sverige, medens der kun kommer en enkelt Elev fra Finland og Island. Til dette skandina- 
viske Omraade var Skolen bestemt, og her fik den ogsaa sin betydelige Indflydelse - trods skiftende Konjunkturer og Resultater. Kun undtagelsesvis møder vi Elever fra selve Tyskland, medens der efter 1864 spores et Tilløb til Søgning fra England. Mere lokalt fik Skolen desuden med Tiden sin store Betydning for Egnen mellem Kolding og Haderslev Fjorde, idet den - trods Konkurrencen fra Skolerne i Kolding og Haderslev - drog ved sin kristelige og pædagogiske Egenart. Man saa saaledes det Særsyn, at Børn fra "de otte Sogne"s Proprietærgaarde ogsaa efter 1864 vedblev med at søge over den nye Statsgrænse til Christiansfeld.

I Skolebyen Christiansfeld mødtes man da med Folk fra praktisk talt Alverdens Lande. Her færdedes stejle Nordmænd, stilige Svenskere, smilende Danskere, flittige Tyskere, tænksomme Hollændere, nøgterne Englændere, iltre Franskmænd, en enkelt Italiener, et spansk Soldaterbarn, og saa de ikke faa verdensberejste fra de oversøiske Lande. Der yar saaledes i denne Klosterby noget at se og at høre fra Jordklodens brogede Folkearena.

Men ogsaa $\mathrm{kulturelt}$ fandt Yderpunkterne Berøring med hinanden paa dette særprægede Sted. Overklassen, Menneskeslægtens kulturelle Top, var — som venteligt — rigeligst repræsenteret. For saa godt som alle dens forskellige Grene var der Repræsentanter til Stede. Der var ikke faa Ætlinge af gamle Adelsslægter, fra Sverige f. Eks.: Snoilsky, Köhler, Fröhlich, Otter, Fock, Posse, Höpken, Krusenstierna, Mannerstråle, Bergenstråle, Dalman, Dahlcrona, Fleetwood, Döbeln, Jägerschöld, Stiernblad o. a., fra Danmark: Lüttichau, Krogh, Stemann, Teilman, Holstein, Holten, Haxthausen, Buchwaldt, Bülow, Rantzau Lehn, Reventlow, Levetzow, Ahlefeld, Wistleben, Schubart, Gersdorf, Kaup o. a.

Andre Elever kom fra førende Handels- og Industrislægter i Skandinaviens Havnebyer, fra Sverige f. Eks.: Keyser, Hülphers, Bager, Björkman, Flensburg, Hedman, Leffler, Plank, Dahl, Ekman, Prytz, Broms, Bagge, Santesson, Zachrisson o. 
a., fra Norge: Collet, Collin, Moe, Valløe, Cappelen, Schjelderup, Kiørboe, Stoltenberg, Hauff o. a., fra Danmark: Hammerich, Tutein, Zeuthen, Drewsen, Esche, Voigt, Baumgarten o. a.

Men ogsaa Læger og Apotekere, Officerer og Embedsmænd, Proprietærer og især Præster sendte deres Ungdom til Opdragelse i Christiansfeld. Af mere kendte Præsteslægter kan nævnes, fra Norge: Hørbye, Crøger, Arup, Wergeland, Bugge, Flood, fra Sverige: Andrén, Rhedin, Silvander, fra Danmark: Ewald, Balslev, Boesen, Mygind, Brandt, Salling, Jeth, Wiberg, Kaalund, Blicher, Plum, Gutfeld, Reiersen, Praëm, Brøndsted, fra Hertugdømmerne: Godt, Kaftan, Valentiner, Grauer, Prehn, Augustiny, Matthiesen, Windekilde, Vent, Neergaard, Ebbesen.

Fantasien faar yderligere Vinger til fortsat Flugt over Aandslivets Verden, naar man blandt Christiansfelds Skoleelever møder Navne, der i Dag er paa alles Læber, fra Litteratur, Videnskab og Kunst saaledes: Heiberg, Marstrand, Ploug, Bloch, Eckersberg, Malling, Lüttken, Rimestad, Østrup, F'orchhammer. Høffding, Zoëga, Ryge, Gebauer, Munksgaard, Salicath, Balle, Stolpe, Fogeklou, Gleerup, Scheel, Horrebow, Gran, Hagerup, Treschow, Groth, Bischof, Wilkens, Drews, Frenssen, endvidere fra Missionsgerningen ude og hjemme: Børresen, Hallbeck, Asboe, Budde, Oxholm, Stockfleth, Koefoed, Steinthal, Riddervold, Solem, Lehmann, Lammers, Malmstrøm, Ries, Lawaetz, Paul Otto Petersen, Bramsen, fra Storpolitikken: Marx, Clodius, Sabroe, Estrup, Hatt, Quisling, Wallenius, Holland, og endelig fra den sønderjydske Grænsekamp: Finnemann, Timmermann, Kylling, Kiil, Staal, Refshauge, Tonnesen, Zachariassen. Eskildsen, Hørlyck, Kloppenborg o. a. Der er saa sandt ingen Mangel paa inspirerende Navne, "fra Vedersø til Jerusalem" og "fra Nebukadnesar til Hitler", selv om de enkelte Bærere her kan være ganske ukendte. Kendte er derimod blevet Camilla Wergeland, Welhavens Tilbederinde, og Riborg Voigt, H. C. Andersens første Kærlighed.

Men over alle disse Kulturlivets kendte Navne bør vi ikke 
glemme Menneskeslægtens Rod, det sunde, friske Blod, hvoraf Kulturen til Stadighed næres, Bonde- og Haandværkerslægterne, der staar paa Grænsen mellem Natur- og Kulturstadet, Ogsaa de var rigeligt mønstrede i Christiansfelds Skoleinstituter, især fra det snævrere Opland Nordslesvig. Hundreder af sønderjydske Mænd og Kvinder har i deres Ungdom her modtaget den Aandens og Hjertets Dannelse, som siden hen satte dem i Stand til at indtage en ledende Stilling i deres Hjemsognes offentlige Liv. Det kan man møde fra Sogn til Sogn Nordslesvig over, fortrinsvis paa Vestkysten. Jeg nævner $f$. Eks.: P. D. Timmermann i Skærbæk, J. P. Petersen i Bredebro, Chr. Finnemann, Taarninggaard, Falle Lildholdt i Løjt, Johanne Høyberg, f. Feddersen i Visby, Bolette Krüger, f. Thomsen i Bevtoft.

Det var i denne Forbindelse uundgaaeligt, at Christiansfeld-Skolerne - villet eller uvillet - maatte blive placeret ogsaa indenfor Grænselandets Nationalitetsk a mp. En saadan folkelig Totalitetskamp paa Liv og Død tillader ingen Neutralitet. Og det maatte da ganske naturligt - efter det 19. Aarhundredes nationale Vækkelse - blive den hjemmetyske Lejr i Nordslesvig, som i denne Skole af tysk Oprindelse og Præg fandt en velkommen Forbundsfælle, medens den til Gengæld mere og mere blev en Torn i Danskhedens vaagne Øje. Der er ogsaa Eksempler paa $\mathrm{Hjem}$, der efter Afgørelsen i 1864 flyttede fra Nord til Syd over Statsgrænsen og sendte deres Børn til Christiansfeld.

Ogsaa religiøst-kirkeligt var der vid Udsyn fra Klosterskolen i Herrnhuterbyen - i Overensstemmelse med Brødremenighedens hele universelle Syn paa kristen Tro og Tanke. Man blev her ikke stængt inde i snævre Bekendelsesgrænser og draget ind i golde Lærestridigheder, hvorved man i Regelen kun styrkes i sin egen Selvtilstrækkelighed. Nej, ogsaa paa dette det dybeste Aandsomraade blev Portene slaaet op for Menneskelivets og dermed ogsaa Kristenlivets sundt individuelle og rigt nuancerede Mangfoldighed. „Hyrden er 
een, og saa hans Hjord, er end fuldmange hans Folde", som Grundtvig efter Evangeliet har udtrykt det, men blandt Kirkeafdelingerne har egentlig kun Brødremenigheden for Alvor praktiseret denne evangeliske Grundtanke. De fleste Elever i Christiansfeld tilhørte - ifølge Omstændighederne - den lutherske Konfession, men der fandtes ogsaa Hussitter, Reformerte, Mennonitter og Katolikker. Ogsaa Jøder fandt Optagelse, af hvilke en Elev, Wera Nürnberg fra København, i 1888 blev kristen undervist og døbt paa Stedet.

Horisonten var - som det ses - aaben og høj over Skolestuerne i Christiansfeld - trods Byens og Menighedens kvantitative Lidenhed og relative Ubemærkethed. Men netop i denne Uforstyrrethedens Stilhed kunde Aandens Skabertime lykkes, kunde der i Opdragelsens Helligdom hentes baade karakterdannende Visdom og kristenpræget Medmenneskelighed. Og Christiansfeld-Idyllen blev utvivlsomt til Daad i adskillige unge Sind gennem Aarene.

$\mathrm{K}$ i l d e materi a le t til Fortegnelsen findes i Arkivet i Christiansfeld, suppleret med Oplysninger fra andre Arkiver. Paa Grund af Christiansfeld-Materialets delvis ret store Ufuldstændighed maa der ved Siden af de aabne Huller ogsaa regnes med skjulte Unøjagtigheder. Af Pladshensyn er der foretaget visse almindelige Forkortelser, som gennemgaaende vil være let gennemskuelige. Pastor Paul Fabricius i Christiansfeld har ydet værdifuld Bistand, naar det gjaldt at gøre Fortegnelsen saa fuldstændig og nøjagtig, som det under de nuværende Krigsforhold lod sig gennemføre. 


\section{A. Drenge:}

\begin{tabular}{|c|c|c|c|c|c|}
\hline Navn & $\begin{array}{l}\text { Fodselsaar og } \\
\text { Hjemsted }\end{array}$ & $\begin{array}{l}\text { Faderens } \\
\text { Stilling }\end{array}$ & Navn & $\begin{array}{c}\text { Fudselsaar og } \\
\text { Hjemsted }\end{array}$ & $\begin{array}{l}\text { Faderens } \\
\text { Stilling }\end{array}$ \\
\hline 1775 & & & .Jon. Ritter & 75 Odense & \\
\hline Johs. Ewald ${ }^{1}$ ) & 60 Hviding & Præst & ('hr. Harpyth & 72 Jylland & \\
\hline ('hr. Heinr. Ewald & 68 Hviding & Præst & Jon. Kramer & 75 Burkal & Soc.A. \\
\hline ('hristl. Mahler & $64 \mathrm{Kbhn}$ & Laerer & Søn. Sønnichsen ${ }^{1}$ ) & 70 Hellevad & Møll. \\
\hline Ole Alsing & 65 Drammen & & Chr. Vothmann & 66 Sønderb. & Grtn. \\
\hline J. C. Ebbesen & 68 S. Bjært & Præst & Andr. Lorentzen & (i5 Flensb. & Kbm. \\
\hline Johs. Hansen & 68 Bjerndr. & Brand. & \multirow{2}{*}{\multicolumn{3}{|c|}{ リ Skoleinsp. i Chrfld. }} \\
\hline J. A. Vieth & 66 Kappel & & & & \\
\hline Johs. Sørensen & 67 (Xliickst. & & 1781 & & \\
\hline J. J. Bæsel & (is Altona & & H. Angel (iude & \multicolumn{2}{|c|}{71 Moss, Norge } \\
\hline ') Soc Arb i liflan & & & H. Sonnichsen & 72 Hellevad & Møll. \\
\hline 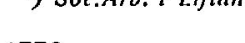 & & & Johs. Sunnichsen & 75 Hellevad & Mall. \\
\hline 1776 & & & ('orn. Adolph' & 70 Nolde & Gidn. \\
\hline Jorg. K. Ham rum & $66 \mathrm{Kbhn}$ & Hskr. & J. G. Hornung & 76 Petersb. & Br.A. \\
\hline $\begin{array}{l}\text { Henr. Balslev } \\
\text { P. Árboe }\end{array}$ & $\begin{array}{l}63 \text { Guldbj. } \\
69 \text { Drammen }\end{array}$ & $\begin{array}{l}\text { Prast } \\
\text { Isage }\end{array}$ & \multicolumn{3}{|c|}{ 1) Missionaer i Surinam 1797-1806 } \\
\hline Jac. Hansen & ¡1 Bjerndr. & Brand. & 1782 & & \\
\hline Hans Lassen & 67 Flensb. & & ('hr. Leth IIolm & $71 \mathrm{Kbhn}$. & \\
\hline F. G. Michaelsen & (i7 Egernf. & & Jens Holm & $7(j$ Khhn. & \\
\hline Joh. H. Thiel & 68 Petersl. & & Ludv. Ritz & $75 \mathrm{Kbhu}$. & I'edel \\
\hline ('xeorg J. Thiel & 70 Petersb. & Br.A. & Johs. Boesen & 74 Nimtofte & Præst \\
\hline F. L. Hornung & 71 Moskva & IBr.A. & (). N. Allelef & 76 Drammen & \\
\hline ('. F. Hornung & 73 Moskvi & Br.A. & $\begin{array}{l}\text { B. H. Santesson') } \\
\text { J. J. Ekman') }\end{array}$ & $\begin{array}{l}76 \text { Göteb. } \\
71 \text { Göteb. }\end{array}$ & Gross. \\
\hline 1777 & & & Gust. H. Ekman & 74 Göteb. & \\
\hline J. Beck Holm & 67 Kbhn. & & H. N. Sonnichsen & 77 Hellevad & Mall. \\
\hline Nils Mïller ${ }^{1}$ ) & (i9) Hjerting & Tldk. & Johs. Kramer & 78 Burkal & Soc.A. \\
\hline $\begin{array}{l}\text { Anor. Warnke-j } \\
P^{\prime} \text {. Henningsen }\end{array}$ & 71 Egerns. & lidm. & 1) Lagge i Gnteborg & $g . \quad$ ) Kommercera & \\
\hline ' Lage i Chrfld. & 3) Soc.Arb. i Stoc & & 1783 & & \\
\hline 1778 & & & J: P. Ingversen & $73 \mathrm{Fr}-\mathrm{ia}$ & \\
\hline A. F. Kiørboe ${ }^{1}$ ) & 65 (Oslo & Khm. & P. Kiørboe & 780 ()slo & Kl)m. \\
\hline Fred. Berner & 68 (islo & & Andr. Fiadda & 75 Oslo & \\
\hline Knud Hansen & 71 Skien & & $\begin{array}{l}\text { Henr. M. Plank } \\
\text { Joh. L. Plank }\end{array}$ & $\begin{array}{l}75 \text { Gøteh. } \\
77 \text { Gøtel.. }\end{array}$ & \\
\hline ') Bestyrer af Hano & delshuset i Chrfld & & Benj. Plank & 79 Gøteb. & \\
\hline 1779 & & & Johs. Dahl & 75 Gøteb. & Kbm. \\
\hline J. J. Bareker & 72 Drimmen & Soc.A. & $\begin{array}{l}\text { Nic. Dahl } \\
\text { Lor. P. Bagge }\end{array}$ & $\begin{array}{l}75 \text { Gateb. } \\
75 \text { Gøteb. }\end{array}$ & Kbm. \\
\hline 1780 & & & (. F. Schonfeldt & 78 Gøteb. & \\
\hline Mich. IIolm & 74 Khhn. & & Andr. Giarling & & Kbm. \\
\hline I'. ('. Rity & 73 Kbhn. & Pedel & Sante Sintesson & 78 Croteb. & Gross. \\
\hline
\end{tabular}




\begin{tabular}{|c|c|c|}
\hline Nic. Schınidt & Bredsted & \\
\hline Joh. N. Schmidt & 74 Bredsted & \\
\hline J. Ren. Plitt & 78 Hamb. & Kbm. \\
\hline J. A. Dïppel & 71 Hrnht. & Br.A \\
\hline \multicolumn{3}{|l|}{1784} \\
\hline J. Brodersen & $79 \mathrm{Kbhn}$. & \\
\hline J. Hallander & 77 Kbhn. & Knt \\
\hline J. G. Høpfner & $72 \mathrm{Kbhn}$. & Bgt \\
\hline P. Wagner & 73 Drammen & \\
\hline Henr. Leffler & Göteb. & Kht \\
\hline Sven P. Lueffler & 76 Göteh. & Kbr \\
\hline Joh. Abr. Leffler & 78 Göteb. & Kbn \\
\hline J. Schønfeldt & 80 Göteb. & \\
\hline .J. Giærling & 79 Göteb. & Kbm. \\
\hline Jac. Giædda & 78 (Gïteb. & \\
\hline P. ('. Ebbesen & 74 ล.. Bjart & \\
\hline
\end{tabular}

1) Moderen Mulatinde

\section{5}

J. .J. ()shek

Johs. Boldt

J. (i. Møller

(i. F. Andersen

79 Fïsberg Præst

77 Tonder Præst

74 Bredsted

76 Slesvig

\section{6}

\section{M. AE. Bugge}

A. Sjöström

Johs. Sjöström

Vilh. Fast

J. W. Eckberg

A. N. Bojsen Johs. Clausen

Nic. Kramer

J. Heinr. Ebers')
79 Bergen

80 (iïtel).

81 Gïteb.

79 Göteb.

76 Høgsbro Gdm.

76 s. Farup Gdm.

80 Burkal Dia.A.

81 Flensh).

Soc.A.
81 Stepping Dia.A.

F. Val. Ritter

89 Flensl).

Fabr.

Jon. D. Schmidt 79 Bredsted

F. Steen Peter'sen 83 Bredsted

1788

Nils Sadolin1)

78 Vive

N. I†. Ellgreen $8 \mathbf{2}$ Göteb.

('. N. Finnemann 80 Dynved Gdm.

Nic. Schmidt 82 Flensh. Kbm.

Heinr. B. Schmidt 82 Bredsted

N. H. Bannier 78 Rendsh.

(. D. Gebauer') 77 Neusalz Br.Pr.

y Prast $i$ Torrild 1826 2) Dyremaler, Prof., $\therefore 1831$

1789

Mich. Røding

Ludw. Dïppel

82 Kbhn. Gross

Hrnht. Br.A.

* Johs Abel

7!) Stepping (idm.

1790

Nils Høyer 80 Aadum Præst

J. G. Kjørboe 8:3 Oslo Kbm.

H. V. Bugge 85 Oslo Soc.A.

(i. v. Dahlerona1) 81 Stockh.

A.S. Winding 83 Höja Præst

P. K. Schmidt 83 Flensb. Klmm.

1) Kancellist $i$ Stockh. ; 1831 af Kolera

1791

F.H. Brøllund 85 Stauning Præst

P. Treschow

Hanson') 83 Laurvig Jern.

J. V. Lejonanker 89 Stockh. Adm.

Isaac Dahl $\quad 82$ Göteb. Kbm.

Peter Dahl 83 Götel. Kl)m.

Daniel Dahl 86 Göteb. Kbm.

Nis Lorenzen 84 Egerns. Tegl.

H. P. Thomsen 81 Kluesgd. Prop.

Jürg. D. Thomsen 83 (Flensb.) Prop.

G. H. Ritter $\quad 85$ Flensb. Fabr.

H. Scharringhausen

86 Chrfld. Soc.A.

1) Larer ved Kadetskole i Osto, Fuldmargtig $i$ Finans-Dep.

1792

Mart. Hammerich 84 Khhn. Gross.

s. H. Røding $84 \mathrm{Kbhn}$ Gross. 


\begin{tabular}{lll} 
Johs. Stengåd & 87 Toarp & Dia.A. \\
Johs. Lejonmark ${ }^{1}$ ) 70 Stockh. & Præst \\
J. F. Silvander & 84 Göteb. & Præst \\
Im. Norling & 84 Göteb. & Bager \\
Sven A. Norling & 85 Göteb. & Bager \\
C. Gust. Norling & 86 Göteb. & Bager \\
(arl Stolpe & 86 Allerum & Dia.A. \\
A. Sønnichsen & 85 Taarning & Møll. \\
K. P. Mygind") & 82 Stepping & Præst \\
\hline
\end{tabular}

i) Hofmarskalk, 2) Postm. $i$ Chrfld.

\section{3}

\section{Christl. Røding}

87 Khlun.
H. V. Høyer

Elef B. Omsted

8õ V. Nebel

sã Drammen

J. S. Jørgensen

\$i skien

Did. S. Jørgensen 87 skien

Erasm. L. Stare 84 Vishy

J. A. Engman 86 (Gøtel).

Isaac G. Sjöström 89 (iötel).

G. A. Henningsen 84 Egerns.

Wilhad. Zoëga

87 Stenderupgd.

\section{4}

Christl. J. Buch 87 Kbhn.

A. Sal. Reiersen 87 Nibe

Lars V. Prytz

82 Göteb.

Josef Gjærling

Elieser Gjærling

Gust. Leffler

86 Göteb.

88 Göteb.

86 Göteb.

Johs. Leffler

89 Göteb.

C. S. v. HIårdt

83 Stockh.

J. G.v. Snoilsky'1) 82 Stockh.

Gust.v.Snoilsky²) 86 Stockh.

J. P. Stolpe

89 Allerum

C. F. Schønfeldt

87 Uddevalla

Chr. E. Schmidt

Jac. Linnich

86 Flensb.

86 Altona

Gross.

Prast

1) Bankass. $i$ Uhyst, +1801 . ") Officer

\section{5}

R. P. Brandt ${ }^{1}$ ) 89 Svendb. Kbm. Jørg. Bang Høyer 89 V. Nebel Præst Lars G. Longberg 82 Stockh.

ソ Fader til Pastor C. J. Brandt.
J. V. v. Iöbeln) 85 Björkh. Gods. Gust. Th. Keyser 85 Stockh. Gross. Johs. Andrèn 87 (röteb. $\quad$ Præst Joh. V. Minten 89 Gïteb. Raadm. Sam. J. Mygind 84 Stepping Prast Lor. H. Schmidt 88 Flensl. Kbm.

1796

Jens Hørbye 87 Krogstad Prov.

Jens Valløe 87 Tonsherg Reder.

Patr. Gegerfelt 86 Göteh. Obst.

G. Wennerstrøm 90 Göteb. Kbm.

J. Wennerstrøm 91 Göteb. Kbm.

Petter Hedman 91 Göteb. Kbm.

C. W. S. v. Man- 86 Jäders- Gods. nerstrále bruk

J. V. v. Dalman 87 Roo Jern.

L. R. v. Dalman 89 Roo Jern.

Dia.A. Jon. L. Winding 89 Engelsh. Gods.

Ed. Th. v. Goes 90 Russel- Gods.

Skom. Joh. C. Ritter 90 Flensb. Falor.

Gdm. Im. B. Thomassen 91 St. Thomas

\section{7}

Joh. Schoustrup $89 \mathrm{Khhn.} \mathrm{Kbm.}$

Hans J. Praëm 89 Øslos Prist

Joh. L. Busch $\quad 86$ Göteh.

Oluf v. Giers 88 Göteb. (Obst.

Andr. v. Giers 89 Göteb. Obst.

Joh. v. Giers 91 Göteb. Olsst.

William Maurice 87 Göteb.

Mismor Maurice 89 Göteb.

Carl L. Prytz 88 Göteb. Gross.

Joh. G. Broms 92 Göteh. Gross.

Jos. O. Norling 90 Göteb. Bager

C. A. L. v. Bergenstråle

Gust. A. v. Bergenstråle 89 Braborg Kpt.

C. A. v. Fleetwood 89 Rynningsh. Baron

M. I. v. Manner- 90 Jäders- Gods. stråle bruk

Fred. v. Manner- 91 Jäders- Gods. stråle bruk

Friedr. Abraham 91 Tønning

H. Dav. Höier 92 Zeist Br.A.

2) Prof. Dr. med., Overfelllage, Halvfatter til
Helten fra den finske Krig 
1798

Mog. C. Muhle

Johs. Jacolssen

J. C. Stoltenberg

E.V. Holmstedt

P. L. Bergman

J. V. Zachrisson

Gust.S. v. Köhler
90 Frholm

92 Skjern

88 Drøbak

89 Philipst.

93 (Göteb.

92 (röteh.

Stockh.

Hojesteretspries.

IJans v. Fock 90 Göthala Baron

('.v. Jägerschöld $90 \mathrm{Karlskr}$.

S.L.v.Jägerschöld 91 Karlskr.

Hinr.v.d.Smissen 87 Altona

Herm.v.d.Smissen 87 Altona

Johs. Linnich 91 Altona

\section{9}

Fr. Haugaard 94 Als .J. Prov. Ove C.J.Reiersen 89 Nibe

J. Green Hørbye 92 Krogstad

Nils A. Nilsen

ole H. Weberg

91 Drammen

Mart. V. Rhedin

93 Drammen

Seb. v. Otter

90 Allingas

90 Hjo

Im. Wennerström 9:3 Göteh.

C. M. v. Krusen-

stierna

Jørg. Ahrnkiel

Lorenzen

Peter Nissen

91 Eksiö

91 Aller

89 Ullerupl.

Johs. Mannhardt

Heinr. Mannhardt 9

* Peter Bugge 89 ()slo

\section{0}

Rasm. Rasmussen 91 Orme-

Soren Kasmussen 93 høj (Fyn)

Hans Hanson

Aug. v. Hárdt

91 Laurvig

F. Wablberg

93 Stockh.

94 Göteb.

J. F. Bergman

95 Göteb.

Carl (). Andrèn

93 Göteb.

Carl C. Sass

91 Hadersl.

Georg Alex. Sass 93 Hadersl.

\section{1}

J. Chr. Jacobsen'1) 95 skjern

P. Abr. Hülphers 91 Stockh.
Ohst.

(obst.

tross.

Giross.

Gross.

Prov.

Prov.

$\mathrm{Kbm}$.

$\mathrm{Kbm}$.

Prov.

Baron

Khm.

Obst.

Mall.

Gdm.

(iods.

Gods.

Soc.A.

Prop.

Jern.

Bnkm.

Prest

Apo-

teker

Dia.A.

(rross.
Lars V. Lindstedt 91 Storkh.

J. F. Sahlefeldt 93 Stockh.

Lor. L. Berger 94 Stockh.

Kh)m.

Arvid I. Berger 96 Stockh. Kbın.

Jørg. Borgström 93 Landskr.

Jürg. P. Todsen 91 Tonder

Krhm.

Jens Hansen

93 Husum

Arrien Wallichs

Jan de Jager

90 W'itzwort

9 Á Altona

Vaver.

Johs. Moehne

9) Gronland

Khm.

Klom.

y Br. Biskop i Amerika

\section{2}

dibhe L. Schough 90 Varberg Insp.

(). J. Wennerholm 9 s (iötel).

E. P. Wennerholm 95 (xïteb.

Joh. P. Hedman 97 (iöteb.

Kóım.

('hr. Moller

94 Hälsingl). Khm.

Jac. H. Kloppen-

$$
\text { borgl) }
$$

P. (). I)insen

Andr. Meyer

94 (iran

'Tngskr.

Laust Nissen

('hr. II. Vent

93 Burkal

(idm.

94 Magstrup (istg.

94 Illerupl. (idm.

92 IIademarschen Prast

\section{C'laes .J. Ney}

92 Altona

Kmd.

Benj. Herhrich

98 Chrfld

Br.M.

') Forf. til ,En gammel Soldals Erindringer" 1803

Thom. Nilsen

C. A. Kullgreen

Drammen Kbm.

J. P. Tönningh

J. U. Mjøberg

Sven A. Broms

Jens A. Beyer

Iddevalla

Hälsingh. Khm.

Giiteb.

(ieorg A. Boysen 94 Tønder Knipl.

(..F. Klentze ${ }^{1}$ ) Altona Prok.

Hinr. de Jager Altona Kbm.

1) Syndikus i Uetersen

1804

P'. C. Simmelkier 9o Helsingmr Hskr.

F. C. Knudsen 95 Borum Prast

J. V.F.croger Hitter- Præst

Georg N. Croger dalen Prest

C. A. Sjögreen 98 Karlskr. 
Jon. Stolpe

Ilans A. Beyer

Claus Müller

P. Wallichs

P. C. Hering

Franz Nielsen

\section{5}

J. M. Gottschalk Jorg. Leth

Iar's Balslev Leth ()tto E. Petersen

Isaac N. Kastberg

A. U. Cronsiöe

Jean J. Cronsiöe

Franz A. Cronsiöe

R. v. d. Lancken

(i. F.v.d.Lancken

Las Danielsen

Peter Meyer

Jens Janniksen

Hen. Henningsen

C.s. Münster

\section{6}

Andr. P. Hørbye

Gust. A. v. Giers

Ephraim Stare

Lars Tönningh

Joh. Peterson

Jürg. F. Jensen

Georg Jessen

H. G. Callsen

Wilh. Glöde

W. Beckmann

J. F. de Saint

Chenoy

1807

Jac. V. Satterup

Andr. Jacohsen

Vilh. E. Weberg

Peter Todsen

Lor.Kamp Todsen 98

Friedr. W allichs

J. H. Pupke
Karlskr. Dia.A.

93 Skærbæk Sgnf.

Kastrup Ldm.

Witzwort Kbm.

97 Altona

97 Altona

Br.Pr.

Ifaurby

93 Svaninge Plirst

97 svaninge Praxst

Kolding

Stockh.

Cronetorp Gods.

Cronetorp Giods.

Cronetory (iods.

Lund Ritm.

Lund Ritm.

92 Skærb)k (idm.

96 Magstrup (istg.

97 Larsbel Gdm.

99 Egerns. (idm.

Altona Pstm.

Sorumgd. Hrrf.

(röteb. Obst.

Göteb. Soc.F.

Hälsingt. Kbm.

slàthult Gdm. (Rigsdagsm.)

93 Tonder Fabr.

95 Tonder Tidf.

Altona

Hamb.

98 Hamb.

Hamb. Port.

02 Kbhn. Klım. Skjern Dia.A.

01 Drammen $\mathrm{Kbm}$.

97 Højer Khm.

$\mathrm{Kbm}$.

Witzwort Kbm. Altona Kbm.
1808

P. Norregaard Khhn. Khm.

Joh. F. v. Leth (O) Odense Kpt.

Laur. Sommer 99 Middelf. Byf.

Jorg. C. Sommer 01 Middelf. Byf.

Term. Hillerup 96 søhygd. Prop.

Nic.smidt Vejle Klom.

Joh. P. Frik 97 Voldberg Moll.

Lor. C. Anderson sverige

Rob. P. Payne Tonning

1809

Chr. Jørgensen Kbhn.

Jørg. Sorteru]) $\left.{ }^{1}\right) 01$ Niestved Vinh.

Holger J. Fahnoe 98 Bogense Khm.

H. v. Lüttichau 04 (irundet. Gods.

Sam. Bentzen 01 Haugd. Prop.

Andr. Raben 98 Bøgeskov Gim.

Chr. Gymoes 95 Feldum Gdm.

Iver Nissen 00 trlerupl. (idm*

Niels Nissen 02 (Nkarhak) Gdm.

Peter Prehn 99 (iliicksb). Præst

J. C.Sievers 03 Altona Khm.

John Hayken (13 Altona Skbf.

W. Beckmann Hamb.

Herm. Hayunga 99 St. Thomas Br.Mf.

リ Jurist

\section{0}

P. Björkman 99 Malmø Khm.

Sam. Björkman 01 Malmø Khm.

Fr. D. Stenbom 99 Toarp Dia.A.

J. C. F. Klemm 03 Altona

Lucas F. Klentze 01 Altona Prok.

Chr. Menzel 04 Grønland Br.M.

1811

(iysbert Miinster Altona Pstm.

\section{2}

Ad. F. Tutein 02 Kbhn Gross.

C. F. Hammerich Odense

Rasm. Nielsen Fyn

W. v. Halling 0:3 Petersh. Prop.

Chr. I. v. Halling 04 Petersh. Prop.

Friedr. Ebbesen 0:3 Hammelev Præst

('hr. R. Moehne') 00 (irønland Br.M.

1) Miss, $i$ Vestindien 181720 
1813

Fr. v. Voss

Sev. Buchwaldt

Heinr. Klentze

Chr. E. Riegen

\section{4}

Niels Buchwaldt 02 Vihorg Skra.

Fred. Springhorg 03 Aalhorg Apo-

J. ('. Springborg 03 Aallorg teker?

Jonas Stare Göteb. Soc.F.

\section{5}

Ebbe J. Buch 03 Vordingb. Farv.

Nic. P. Buch 08 Vordingl). Farv.

S. Chr. Brandt 116 Svendb. Kbm.

Heinr. Hammerich

P. A. Rasmussen

Joh. C. Bau

Peter Bager

Isaac A. Ståhle

Peter F. Ståhle

P. Möller

(ieorg Hayunga

Peter Friestedt

\section{6}

Carl R. v. Holten

H. J. Hillerup

T. R. v. Teilman

Lar's F. Gram

Wilh. Klentze

Lor. W. Riegen

Rob. Mc.Cormick

John Me.Cormick

\section{7}

Ernst C. Ludwig A. F. Brøndsted Friedr. v. Krogh Jul. v. Steman

J. C. Eskildsen

M. I. Klentze

Fr. J. Weinmar

\section{8}

Jul. Lund

P. J. Haagesen
Odense

Bramstr. Prop.

Vanse Dia.A.

Catrinetorp Kbm.

Hälsingb. Brgm.

Hälsingl). Brgm

Hälsingb. Kbm.

St. Thomas Br.MI.

St. Jan Lndf.

06 Odense Et.R.

02 Bramstr. Prop.

05 Endruph. Gods.

Hälsingb.

Altona Prok.

Altona Kbm.

(St. Croix Magl.

|(Flensb.) Mægl.

Klohn. Slagt.?

07 Varde Præst

08 Taabdrup Gods.

Hadersl. Obst.

07 Runde Møl. Møll.

Altona Prok.

St. Thomas Br.M.

Kbhn.

Kbhn. Skom.
A. P. Andersen

Khhn.

W. C. Andersen 06 Sorø

Jørg. Nielsen 12 Flensh.

('hr. Tanck1) Rendsb.

Fr. J. Grillich 06 Gronland Br.M.

') Lage i Tonder?

\section{9}

Fr. Jørgensen

Kbhn.

'Theod. C. Ross 09 Kbhn. Kbm.

Peter Petersen Kbhn. Skom.

Chr. Matthiesen $10 \mathrm{Kbhn}$. Gross.

Hartv. v. Linstow 10 Frydenl. Prop.

Ax.J.v. Stiernblad 10 St. Markie Baron

Fred.v.Stiernblad 10 St. Markie Baron

Will. Weinmar 11 St. Thomas Br.M.

\section{0}

Carl E. Friis $\quad 06$ Khhn.

Peter .J. Hois $10 \mathrm{Khhn}$ Khm.

Ludw. Klein $10 \mathrm{Kbhn}$. Bager

Carl Klein $\quad 11 \mathrm{Kbhn.}$ Bager

Ferd. Klein $13 \mathrm{Kbhn}$. Bager

J. Chr. Gebauer 1) $08 \mathrm{Kbhn}$. Dyre-

Carl Gebauer $08 \mathrm{Kbhn}$. maler

Thomas Selmer 13 Hørsholm Ju.R.

'Term. Fabricius 0s Ansager Præst

Erich G. Warnke 11 Stockh. Soc.A.

' Komponist, Organist ved Helligaandskirken, 1884 .

\section{1}

Aug. Esche

Kbhn. Gross.

Vald. Ryge

Kbhn. Sksp.

Andr. E. Baul) 15 Vanse Dia.A.

Hans Nicolaisen 10 Logumkl. Glrm. Ed. Wright $\quad 16$ St. Thomas Br.M.

り Miss. i Surinam 1852-77

\section{2}

Jul. Esche

C. F. Schumacher

Kbhn.

(iross.

Wilh. Gebauer

Khhn.

H. J. Hansen

Kbhn.

Maler

Carl Göransson

$17 \mathrm{Kbhn}$.

Stockh. Apo-

Rudolf Güransson Stockh. teker?

Jul. v. Stiernblad 13 St. Markie Baron 
1823

Conr. Wallis

Em. Steinthal

Ferd. Hoyer

\section{4}

(arl S. (irønlund 12 Lundum Prov.

\section{5}

Joh. P. Bager

Lor. Th. Bager

Aug. Hoyer

18 Malmø

Kbm.

19 Malmø

19 St. Jan

Kbm.

Br.MI.

\section{6}

('. ('hristiansen') $16 \mathrm{Kbhn}$.

Vilh. F. Herre

Sam. Hofman

Elias M. Bau')

Lor. I. Bager

$\begin{array}{cl}\text { Oslo } & \text { Gross. } \\ \text { Chr-sund } & \\ 19 \text { Vanse } & \text { Dia.A. } \\ \text { Malmø } & \text { Kbm. }\end{array}$

C.Ambd. Brorson 19 Nordb. Styrm.

3) Kbm. i Petersb., gift med Datter af Pastor Roentgen i Chrfld, ') Miss. $i$ Surinam $1819-61$

\section{7}

Vald.v.Stockfleth 15 Kolding

Carl Nllsen

Oluf Roll

Drammen Kbm.

Trondh. Ass.

\section{8}

O. v. Haxthausen 21 Oslo

Ole Taarvig

14 Arendal

C. Forchhammer

Ed. Rollwagen

14 Tyrstrup Præst

1829

Em. Wissing

Carl Th. Wissing

Peter Collet

G. Ed. Dahl

J. Fred. Dahl

('hr. L. Marsch

\section{0}

Heinr. Zuvan

Andr. Krogness

Gust. Warnke

Chr. Lehmann

\section{1}

Mich. I)ahl

Jac. Dahl
14 Reval Br.A.

$\begin{array}{rr}\text { Kolding } & \text { Kbm. } \\ \text { Kolding } & \text { Kbm. } \\ 18 \text { Oslo } & \text { Kbm. } \\ 16 \text { Göteb. } & \text { Kbm. } \\ 19 \text { Göteh. } & \text { Kbm. } \\ 16 \text { Skast } & \text { Gstg. }\end{array}$

17 Oslo

Trondh.

18 Stockh. Soc.A. Gronland Br.M.

19 Holstebro Prop. 21 Göteb. Kbm.

\section{2}

(Keorge v. Deurs's) 25 Nakkebølle Prop.

1) Mis.s. $i$ Vestindien 1852-5.3

\section{3}

C. P. A. Basberg 20 (Oslo

Thure C. Norling 24 Stockh. Dir.

Alb. Wallis 24 Stockh. Gross.

Ephraim Bager') 24 Malma Kbm.

Jürg. Marcussen 20 Aabenraa Orgb.

Niels L. Ocksen 21 Skærbæk Gstg.

Johs. H. Petersen $19 \mathrm{~V}$. Alslev (idm.

Jac. J. Plaettner 27 St. Croix Br.M.

Joh. A. Plaettner 29 St. Croix Br.M.

\section{1) Prast $i$ Tygelsjo, Dr. phil. 1847, Provst 1972, dod 1090 (jfr. J. Hartler $i$.,Lunds stifts julbok 1942 , Side $(-20)$}

\section{4}

Kai Schumacher 18 Hadersl. Lage

Antoni Beck 19 Gravesl.

* Valentin Ritter 24 Flensb. Ldm.

1835

Fred. Brandt 25 Trondh. Lt.

1836

Corn.A.Sjökrona1) 24 (Gustafsl. (). Jæ-

Hans A.Sjökrona 25 (Hailsingh.) germ.

Peter N. Marsch 22 Skast Gistg.

Otto D. Marsch 23 Skast Gstg.

J. Gr. Mehlhose 27 Gronland Br.M.

Fr. W. Mehlhose 30 Gronland Br.M.

Fr. Münzer 29 Antigoa Br.M.

* Wald. v. Bielke Chrfld. Forp.

1) Generalmajor, Ejer af Helgena's

1837

Henr.P.Tranberg 25 Varde Kbm.

Carl J. Th. Bock 23 Witzegd. Prop.

Ldw.P.C.R.Bock 27 (Aalb.) Prop.

Em. Winter 27 Fr-hald

Theod. Radich 23 Fr-hald Kpt.

Carl .J. Lund 24 Fr-hald Kbm.

C.M.Matthiesen $\left.{ }^{1}\right) 20$ Oversø Præst

C. G. Lorenzen 25 Refsø Gods.

Jul. Renkewitz 31 Jamaica Br.M.

) Arkivar i Rigsarkivet 
1838

Guido Brandt

Ludv. Hammar

(C. E. Hedström

Jürg. C. Petersen

('arl Friis

(rottl. Marseh

Haders

25 skast

F.M. Matthiesen 1 ) 23 Overso

H. Windekilde

26 Dorpat

28 Hapsal

31 (Lifl.)

Ed. A. Werner

1) Fader til Past. H. I. F. C. M., sidst i Hadersl.

\section{9}

Victor Müller $28 \mathrm{Kbhn}$.

Thorv. H. Vallae 28 'Tonsb. Reder

Herm. A. Valloe T'onsh. Reder

And.Valeurvalløe 30 Tonsh.

l'eter Bager 24 Malma

Hans H. Petersen 26 Dalhy

Johs. H. Petersen 27 Dalby

Ed. v. Simesen 26 Hadersi.

(. F. Thraen

31 Antigoa

John Münzer

33 Antigoa

Fr.Th. Renkewitz 33 Jamaica

*.J. D. Johansen 24 Vonsild

* Ernst Ritter

29 Flensh.

1840

$\begin{array}{lrr}\text { H. H. Laffrenz } & 33 \text { Horsens } & \text { Klkt. } \\ \text { Peter Blom } & 28 \text { Drammen } & \text { Skbf. } \\ \text { Stevelin Irdahl } & \text { Fr-stad } & \text { Skbf. } \\ \text { ('hr. Togesen } & 27 \text { Stenderup } & \text { Ldm. } \\ \text { P. Benn. Hansen } & 26 \text { Aabenraa } & \text { Kbm. } \\ \text { John M. Mudie } & 31 \text { St. Croix } & \text { Forv. } \\ \text { William A. Mudie } & 32 \text { St. Croix } & \text { Forv. }\end{array}$

1841

$\begin{array}{lll}\text { C. F. Paulsen } & 29 \text { Kjolstrup } & \text { Prop. } \\ \text { H. O. S. C. ( Gross } & 27 \text { Kolding } & \text { Tldk. } \\ \text { (1. Ferd. Tranberg } 27 \text { Varde } & \text { Khm. } \\ \text { Jul. ('hristensen } & 30 \text { Lemvig } & \text { Sagf. } \\ \text { Ed. Thomsen } & 27 \text { Trondh. } & \\ \text { Jens R. Valløe } & 25 \text { Tonsberg } & \text { Reder } \\ \text { N. Fr. Hauff } & 27 \text { Sandefj. } & \text { Knsl. } \\ \text { Johs. Hansen } & 26 \text { Hadersl. } & \text { Iarer }\end{array}$

M. H. Mathiesen 26 Bylderup (idm. Em. Ihrer 35 Gronland Br.M. * (aj W. Salling 30 Vonsild Prast 1842

H. Rübner Møller 27 Konst-borg Prop. N. Kübner Møller 30 (Aarhus) Prop. H. A. Valløe 30 Tønsl. Reder Andr. Staude 28 Hamb. SlagJul. F. ('. Staude $31 \mathrm{Hamb}$. ter

\section{3}

.Johs. Lange 30 Rødkilde Crods. Palle H. Elkiar 28 Vellinggd. Prop. Jens (. Aamodt 30) Chr-sand Tldk. Joh. C. Rasch 30 Drammen S.Skkr. Aug. (f. Flood 35 Trondh. Præst Th. H. Schlytter 30 Laurvig Magl. G S. Jacobsen $33 \mathrm{Fr}$-hald

G. J. (ileerup 28 Lund Bogtr. S. P. A. Björkman 29 Malmg Kbm. V. Th. Bjorkman 30 Malmø Klom. J. A. Haastrup 31 Ødis Gstg. Chr. Tonnesen 28 Stenderup Gdm. Jannik J. Beyer 30 Barsbøl (xdm. Chr.k. Beyer 32 Barsbol Gdm. * (reorg Ribbins Antigoal

\section{4}

Joh. C. Arneberg 32 Vanse Præst Fr. Hauf Valløe 32 Tønsb. Reder Lars Westergaard 28 Arendal Kibm. C. Joach Mohn 32 Bergen Klom. Ole Throness 30 Chr-sund Cl. N. Helsing 30 ( hr-sund (. Th. Dahl 1) 30 (iïteb. Khm. Cr. Rasinussen Hadersl.

P. B Neumann 28 Vilstrup (idm. Herm. Klentze Altona Prokur.

* Peter Schiøtz 27 Stavanger By-

* Soren D. Schiotz 28 Stavanger foged

* IT. C l3oesen 28 Vordingh.

リ Miss. i Surinam 1866-89. 1845

Ad. v. Hein 3s Bygholm Propr. P.olivarius Flood 29 Trondh. Prest Elias flood $\quad 37$ Trondh. Prast 


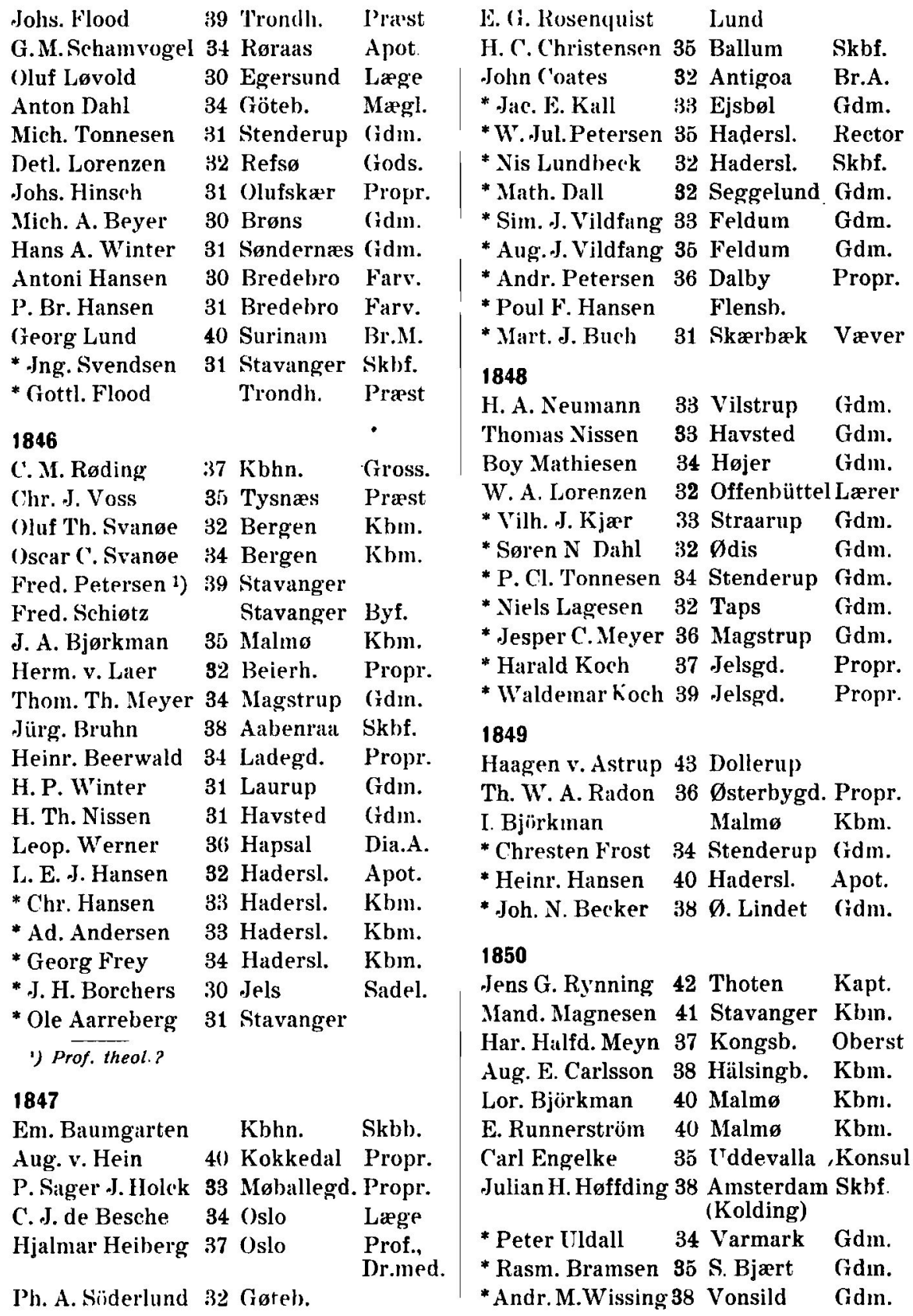




\section{*'Jul}

Ludv. E. Iversen 41 Dorthesm. Propr. Fred.Freiesleben 37 Aarhus Oberst

Lars Berentsen 38 Stavanger Kbm.

F. A. H. v. Bildt 36 ITdevalla Khm.

Carl F. Signeul 41 Karlsham Kontr.

C. (). Th. Sylvan 38 Ystad Domm.

Bror A Sylvan 39 Ystad I)omm.

J. H. E. Dieden 40 Malmø Khm.

Otto V.v. Bennet 40 Malmø Major

Corn. H. F. Roll 35 Hadersl. Lage

('aspar Nissen 35 Hadersl. Rebsl.

P. A. (Yöhlmann 35 Hadersl. Garv.

.J. G. Göhlmann 41 Hadersl. (Yarv.

Wilhad. B. Ernst 41 Hadersl. Hatt.

Heinr. Nissen 43 Hadersl. Kbm.

Peter Neumann 35 Vilstrup (idm.

Jes. P. Neumann 35 Vilstrup (idm.

Iver B. Holm 36 Vilstrup (idm.

P. C. Christiansen $3 \overline{0}$ Siverkro Gastg.

('aspar F.S.Dircks 37 Storkseehof

F. H. G. ()tte 44 London Skom.

Georg Zschoch 45 Petersb. Br.A.

Th. Renkewitz 44 Jamaica Br.M.

* C. Volquardsen 40 Hadersl. Idm.

* P. Volquardsen 43 Hadersl. Ldm.

* Eug. v. Laer 38 Beierh. Propr.

\section{2}

\section{Tage A. Sylvan}

35 Ystad

E. I. Flenshurg

.Joh. Dall

Nis P. Biehl

\section{Malmø}

37 Seggel.

37 N. Hjarup

And. P. Lund 1)

Jul. M. Lund

42 Ballum

42 Grønl.

4ล̆ Grønl.

* Jac. Østergatard 37 Grønholt

*'Truels J.Schmidt 36 Seest

* HeinrA. Paulsen 39 Tønder

* Nic. Traustedt $3 \times$ Løjtved

* H. P. Petersen 36 Ølgod

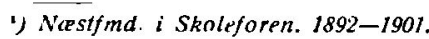

\section{3}

Mart. V. Alhinus 43 Kolding Kane.R. Jens Simonsen 39 Ravnholt (ydm. Jeppe ('hr. Bank 41 Gimsing Gdm. Reinh. Magnesen 43 Stavanger Kbm. Fritz E. Follin 42 Posiöh Ritm. Peter Kjerrulf 40|Præst- Bruks(x. F. J. Kjerrulf 42/hytte patron LarsG. Osterberg $40 \mathrm{Chr}$-stad Bygm. P. .J. Wissing 40 Vonsild (ridm. C. Th. Lassen 39 Bjerndr. $\quad$ (rdm. II. H. Neumann 37 Vilstrup $\quad$ ( $\mathrm{Idm}$. H. H. Posselt 41 Frorup Gdm. P. J. Jangaard 38 Tapsur (idm. Mart.S. (Yronnet 38 Bastrup (idm. Jac. Matzen 39 Løjtved Sunf. Jørg. A. Petersen 38 Bredebro Bager Jens l'. Petersen 40 Bredebro Bager Jonas Asboe 47 Gronland Br.M. *C. E. Andersen 46? Hadersl. Khm.

*Axel (arstens ${ }^{1)} 47$ Borum Prast

* Hans I. Staal $\stackrel{1}{ } 38$ Tatarning Møll.

* Soph. F. Kjar 38 Straarup Gdm.

- Otto A. Kjær 40 Straarup Gdm.

- Fritz H. Kiæl 43 Strairup Gidm.

* Peter Knuth Flensb.

1) Tandlage, Fmd. for de samvirkende sonderjydske Foreninger. "2) den bekendte Medstifter af Skamlingsbanke-Selskabet.

\section{4}

(hr. S. Bøving

Søren Buus

('arl F. J. Fríck

Niels .J. S. Silow

Sam. F. Hedman

Laust Thygesen

Hans B. Gram

P. C. Nissen

('hr. P. Hansen

(Teorg Andersen

H. Mertelmeyer

Edm. ('hild

F. L. Meynell

- Bertel H. Dahl
41 Egtved Prast

40 Ørumgd. Propr.

40) Malmø Kbm.

42 Malmø Kbm.

43 Göteb. Kbm.

40 Ballum $\mathrm{Kbm}$.

41 Ballum Skbf.

39 Havsted Gdm.

38 Hjolderup Gdm.

41 Tyrstrup Forp.

45) Luibeck Vinh.

36 Wanstead

42 Engl.

tis Talarning Gdm. 
*('hr.Finnemann1)42 Taarning Gdm.

* Gust. Petersen 41 Hjerndr. Gdm.

') udvist 1902-07 (Sprogforen. Almanak 193I, S. $70-73)$.

\section{5}

Mart. J. Schmidt 39 Lundersk. Gdm. Ilans Schou 42 Saby Khm. P. A W'issing 42 Kolding Khm. Oluf Lea 41 Stavanger Garv. Elias Due 45 Stavanger

Aug. E. Dyberg (i. F. Björkman Jac. C. Kull 42 Malmø Kbm. 47 Malms Kbm. 44 Quiddinge Kantor Chr. Ang. Lalssen 42 Bjerndr. Gdm. H. Rostgaard

$$
\text { Evald }
$$

Andr. Lorenzen

40 Højrup Gdm. 39 Kinud Gastg. $\$ 1$ Bræendur Moll. Chr. Iversen Lund 41 Brondlund Gim. Rich. S. Jobson Corn. Heiliger 41 Scottl. Ldm. 49 St. Croix Br.A. Dav. Marx

* Vald. Kjar

* Axel Kjær 40 New York Br.A.

4i) Straarup (idm.

45) Stratap Gdm.

* Jens Raben 42 Tyrstrup Gdm.

* Mads E. Nielsen 42 Hjerndr. Ldm. * Jesper Clausen 44 Harreslev Gdm.

\section{6}

Hans .J. Berg 42 Fr.havn Kbm. Jac. R. Hald 44 Keldby Provst Carl C. Wolff 42 Herningh. Propr. Jac. E. J. Walther 46 ( $\mathrm{C}$ hr-sund Kbm. Hans M. Moe 45 Chr-sund Prit. Ludv.A.Hatteberg 45 Bergen Soph. L. Sticklers 47 Malmø Math. Flenslourg 43 Malmo Rud. F. E. Planius 44 Chr-stad Oscar A. Planius 46 Chr-stad C.J.H Schwieler 45 Stockh. H. A. Ocksen 42 Skærbæk E. L. Timmermann

Jens Jensen

44 Skxrbak

Marcus Neumann 40 Vilstrup Jacob H.Neumann 43 Vilstrup
Bendix Mommsen 41 St. Tonde Gods. H. ('. J. Todsen 4ó Meierh. Propr. PoulO.Petersen ${ }^{1}$ ) 44 Højer E. G. J. Fürsen 40 Leck Gdm. Osear C. Clason $40 \mathrm{Hamb}$. Fabr. Louis IIansen $\quad \mathbf{4 2}$ Lübeck $\mathrm{Kbm}$. Ditlef $H$. Lorenzen 42 Offenbüttel Lærer Herm. Jannasch 49 Syd Afrika Br.MI. * C. Arnold Juhl 4\& Tyrstrup Læge

* Andr. Andersen 49 Tyrstrup Forp.

* Jeppe H. Krog 40 Vejstrup Gdm.

* Joh. Paulsen 41 Brahæk Møll.

1) Miss. i Tirupati $1875-88$.

\section{7}

Chr. V. Kyhl 42 Julianeb. Propr. Fr. Theod. Kyhl 46 Julianeb. Propr. P. Seest Boesen 45 Petersm.] Propr. Gust. Gundersen $43 \mathrm{Chr}$-sund Skræ W. M. Westrell 45 Malınø Kbm. A. H. Göransson 43 Gefle Konsul ErnstF.(iöransson 45 Gefle Konsul John N.Göransson 46 Gefle Konsul Chr.J.v. Rappe 43/Strøms- Baron Erik N. Rappe 45 rum Baron Jonas R. Bagge 43 Hernüsand Bruk. Peter Beyer 44 Barsbøl Gdm. Chr. Carstensen 41 Rørkær Gdm. Andr. Toft Car- 43 (Tønder) Gdm. stensen

Jens Seneberg 43 Hadersl. Bogtr. Jac. Horshøll 42 Langetved Gdm. Woldem. Garve 43 Altona Br.Pr. Reinh. Garve 46 Altona Br.Pr. Victor E. Pallisen 44 Petersl).

* Niels P. Brandt 50 Bjerndr. Husm.

* Mich. Michaelsen 41 Stavanger Garv.

* Rasm. Michalsen 42 Stavanger Garv.

\section{8}

Niels C. Sabroe 47 Alstedgd. Propr. Oscar Brusewitz 44 Göteb. Kæm. (iust. H. Carlsson 47 Malmø Mrgl. Gust. V. E. Berg 48 Karlsham Kbm. P.H. Petersen 47 Binderup Gdm. 
P. Degn Timmermann ${ }^{1}$ ) 46 Skærbæk Gdm. Jøst G. Netlohes

* Fr.'Erdmann

) Jfr. "Haabets Mand", S. 233-38 (1923).

\section{9}

L. K. Kaspersen 49 Kbhn.

C. Estrup Hansen 49 Odense

O.F.C. Sørenson 51 Vejle

L. M. Svendsen

H. Isaachsen

W. Spechmann

Erik A. Hallbeck

C. R. Björkman

P. Gerh. Ovenius

Arvid Hultgren 48 Mariastad

F. V.Österberg $44 \mathrm{Chr}$-stad

Andr. P. Eeg

P. P. Vesterbye

P. M. Petersen

Gust. W. Holst

Jos. Thompson

* O. Val. Juhl

* Jac. Michaelsen

* J. H. Lund

* Peter Brandt

1860

Carl Høegh

Sam. H. Trennery 47 Trondh.

H. P. Wolter

Peter Eggen

Hans E. Halse

Oluf Halse

Em. Ringheim

John Martinsson

Gotth. Dieden

Barthold Dieden

Carl Peters

Henr. A. F.Peters

C. Gust. Fock

Otto Tullberg

Theod. Petterson

Carl Nissen

Kaj H. Lind

43 Skartved

43 S. Bjært

44 Bredebro

47 Graasten

46 New York

50 Tyrstrup

Kbm.

Kbm.

Kbm.

Kbm.

Sadel.

Fabr.

Kbm.

Konsul

Bygm.

Gdm.

Gdm.

Bager

Gods.

51 Chrfld Dia.A.

Б0 Bjerndr. Husm.

Math. Holgersen

\section{Trondh.}

Læge

53 Trondh.

Trondh

48 Trondh.

52 Trondh.

45 Karlskr.

47 Malmø

48 Malmg

49 Malmø

$4 \check{5}$ Stockh.

48 Stockh.

46 Lidköp.

47 Lund

46 Lund

49 Torreberg

49 Vonsild

47 Halk
Værkm.

Soc.A.

Garv.

Garv.

Kptlt.

Fabr.

Fabr.

Regi-

strator

Kpt.

Provst

I'.off.
Evans Willerup

* Alb. v. Bülow

\section{1}

Carl A. Bock

Nic. Løkke

C. J. Burchardt

A. Th. Grønwall

Fr. H. Flensburg

Oscar H. Silow

Carl H. Ovenius

Otto H. Collin

Ed. J. Wiberg

Ax. F. Ritter

Greg. Jorgensen

Jorg. A.Jørgensen

Mik. Uldall Juhl

Ed. Huesmann

Andr. Asboe 1)

W. Jannasch

Lewis Hassé

* Laur. H. Klyhn

* Tøn. Michaelsen

y) Miss, paa Grenland 1884-88.

1862

Ephraim Wolter

Gust. Aarøe

Carl Aarøe

C. Th. Oxholm

$54 \mathrm{Kbhn}$.

53 Kbhn.

Kbhn.

Fr. v. Arenstorff

Carlv. Arenstorff 51 Overgd.

L. N. H. Lorenzen 48 Clasonsb.

Johs. Stephansen 48 Yttren

Joh. P. Collin 49 Sölvitsb.

Bror Jesper Collin 50 Sülvitsh.

Sven O. Collin

53 Sölvitsb.

Knut E. Collin

54 Sölvitsh.

Herm. Th. Müller 48 Malmø

Joh. P. J. Hintze $49 \mathrm{Chr}-$ stad

Carl Lōnblad

$53 \mathrm{Chr}$-stad

Gust. E. Holm

Pehr J. Bagge

C. Fr. Bagge

Joh. Kullmann

Chr. Balle
47 Chr-stad

49 Kramfors

49 Kramfors

47 Näsby

47 Hejls
Kpt.lt.

Audit.

Khm.

Khm.

Konsul

$\mathrm{Kbm}$.

Ǩbm.

Insp.

idm.

Gdm.

Gdm.

Br.M.

Br.M.

Br.Pr.

Skilosf.

Garv.

Soc.A.

Pr.lt.

Pr.it.

Khm.

Propr.

Propr.

Propr.

Kbm.

$\mathrm{Kbm}$.

$\mathrm{Kbm}$.

Kbm.

$\mathrm{Kbm}$.

O.læge

Kond.

Insp.

Insp.

Gidm.

Gdm. 
J. Rich. Petersen 48 Olufskær Johs. Danielsen 47 Fredsted E. Jul. Danielsen 49 Fredsted Lvelin R. Hassé 55 Gracehill

Propr. Propr. Propr. Br.Pr.

\section{3}

H. Chr. Ferslev Jac. H. Werner

54 Horsens

51 Chr-sand Seth A. Franzén 50 Umeá Aug. Österberg $48 \mathrm{Chr}$-stad Henr. Th. Wilkens 49 Karlskr. Giithe Th.Wilkens 40 Karlskr.
C. Wilh. Ek

49 Lund

Andr. Petersen

H. C'hr. 'Thomsen

Nils Thomsen

Ludv. Ruge

Chr. Jürgensen

P. A. Jensen

Adolf Nissen

Peter H. Toft

Sam. Glöckler

* Asmus Leu

* Wilh. Leu

* H. II. Holm

* Anton K. Lund
48 Dalby

50 Skodborg

49 Hjarup

55 Hadersl.

50 Hadersl. Hadersl.

49 Havsted

48 Hornse

51 Surinam

49 Taarning

62 Taarning

50 Ulstrup

47 Stavanger Skbf.

\section{4}

Math.S. Wolter $\left.{ }^{1}\right) 56$ Kbhn. Soc.A. Andr. W. Holck 52 Horsens Propr. Fr. Münchmeyer 50 Marieholm Gods. Heinr. Lorenzen ō1 Refsø Gods. Jørg. H. Jørgensen 50 Stenderup Gdm. Chr. Petersen Knud P. Dahl Niels Chr. Astooe o0 Dalhy Propr. 49 Taarning Gdm.

* Johs. Simonsen

* S. Th. Simonsen

* P. D. Paulsen 56 Grønland Br.M. 54 Tyrstrup | Vejo5 'Tyrstrup J insp.

* C. v. Ahlefeld 65 Skovrup Snedk.

* L. v. FischerBenzon 54 Chrfld. Postm.

* W. H. Wriedt

56 Chrfld. Tldk.

* L. Berthelsen o5 Chrfld. Tldk. 50 Arrild Gdm.

\footnotetext{
1) Miss. $i$ Vestindien 1894-1926.
}

\section{5}

A.J.H.Bergendahl $61 \mathrm{Chr}$-sand Gross.

T. W. Bergendahl 52 Chr-sand Gross.

K. A. Bergendahl j4 Chr-sand Gross.

Carl L. Halén $\quad \overline{5} 2$ Hernösand Gross.

Falle Tr. Uldall 49 Binderup Gdm.

Mads N. Have 51 Vonsbæk Gdm.

Franz Hinrichsen 51 Kollund Gdm.

Peter Hinrichsen 54 Kollund Gdm.

* Otto Valentiner 54 Tyrstrup Præst

* Max Valentiner 56 Tyrstrup Præst

* Jos. v. Neergaard 52 Aller Præst

* Tr. Neergaard 53 Aller Præst

* E.Schwerdtfeger 54 Bramdrup Gdm.

* Chr. Asmussen 51 Tyrstrup Gdm.

* Johs. Lehmann 51 Tyrstrup Slagt.

* Chr. Lehmann 56 Tyrstrup Slagt.

* Th. C. Møller 49 Kappel Guld.

* Carl Herbrich 56 Grønland Br.M.

* C. E. van Laer 64 Auburn (Am.)

\section{6}

Hans Jørgensen 55 Ferup Gdm.

P. Neerg. Madsen 56 Grumstrup Gdm.

H.O.F. Kunig 54 Trondh. Tømr.

Jeppe N. Juhl 51 Skartved Gdm.

Math.S.'Jørgensen 52 Stenderup Gdm.

Georg C. Petersen 53 Dalby Propr.

Herm. Dehm 57 St. Croix Br.M.

- W. v. FischerBenzon

58 Chrfld. Tldk.

* Fr. Valentiner 58 Tyrstrup Præst

* Fr. Bohnfeldt 50 Fjelstrup Sgnf.

* P. C. Fallesen 61 Knud Gdm.

* Iver Lorenzen 51 Knud Gdm.

* Laur. Thorøe 50 Anslet Gdm.

* J. J. Jensen 52 Brabæk Gdm.

* Heinr. Rudebeck 57 Tyrstrup Husm.

* Carl Lorentzen 51 Hadersl. Kbm.

\section{7}

Mart. Albrethson 57 Tromsø Skbf.

Gust. I. Silow 53 Malmø Kbm.

Gust. Møller 50 Göteb. Kbm.

L. A. W. Meyer 51 Lund |Adj.

Ernst G. H. Meyer อ̄6 Lund |Dr. ph. 


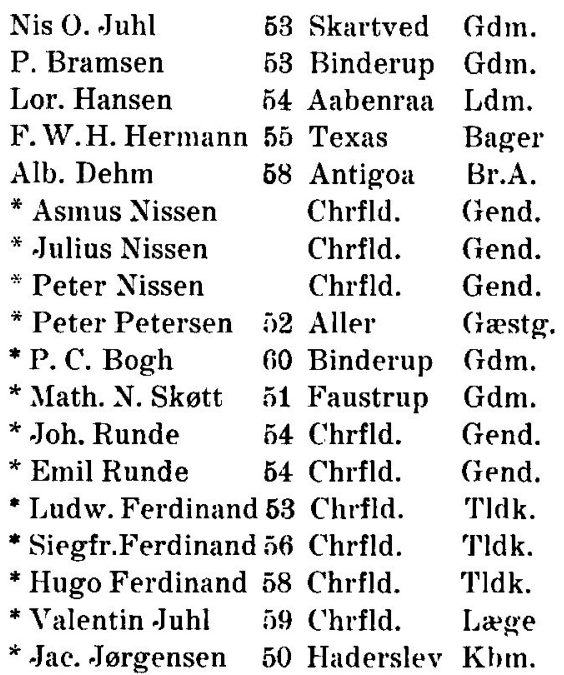

\section{8}

Bertram Hegge

C. Christiansen

Laur. O. Juhl

Nis G. Lind 1 )

F.Hinr. Feddersen 5:3 Tømmesh. (idm.

ITde Thaden

Ethelb. Saunders

* Carl Jørgensen

* H. Andersen

* Jeppe Barsøe

* Laue Friis

* Marius Madsen

* Nils Liin

* Mart. Boysen

54 Trondh. Hatt.

5i) Trondh. (Yrastg.

54 skartved Gdm.

5 S. Bjært (idm.

52 Arnis

56 London

53 Brændur

52 Aller

Moll.

7 Aller

55 Stubbum Gdm.

53 Aller Degn.

59 Aastorp Gdm.

53 Løgumgd. (irdm.

56 Tøstrup I'rm.
1) Kontorchef $i$ Kbhn.

\section{9}

Chr. Th. Wolter') 61 Kbhn. Soc.A. Laur. Bramsen 58 Stenderup Propr. Chr. Petersen 54 Hadersl. Khm. Alfred Raben $\quad \overline{0} 4$ Hadersl. Tldk. Aug. OhIsen 55 Stendetg. (idm. Martin Adolf 56 Vollerup (idm. .Jes. P. Brodersen 54 Ulemberg ( $\mathrm{rdm}$. Otto Lück 56 Meldorf $\mathrm{Kbm}$.

$\begin{array}{lrl}\text { Ernst Wilde } & 55 \text { Lübeck } & \text { Kbm. } \\ \text { Harry Isitt } & 53 \text { Bedford } & \\ \text { * Jørg. P. Have } & \text { Brabæk } & \text { Gdm. } \\ \text { * Andr. Bruhn } & 54 \text { Seggel. } & \text { Gdm. } \\ \text { * Chr. Jørgensen } & 55 \text { Brændur } & \text { Møll. } \\ \text { * Adolf Jørgensen } 56 \text { Brændur } & \text { Møll. } \\ \text { * Ferd. Jørgensen } 59 \text { Brændur } & \text { Møll. } \\ \text { * Johs. Bogh } & 57 \text { Binderup } & \text { (jdm. } \\ \text { * Chr. Juhl } & 62 \text { Chrfld. } & \text { Lage } \\ & \end{array}$

\section{0}

J. H. Bjerrisgaard 54 Egum (idm.

Just. Justesen $5 \bar{n}$ Krathus (idm.

Johs. Tippmann 61 Trondh. Soc.A.

Clemens Coumbe 5ñ London

Sidney Hartman 58 London

* C. v. Ahlefeld 64 Chrfld. Postm.

* Artl. Ferdinand 63 (Chrfld. Tldk.

* P. Lauritzen $57 \mathrm{Fr}$-ia

* Fritz Martens 62 Husum

1871

Nicolai Hiis $\quad$ i7 Arendal Skbf.

Mart. Gundersen 58 Arendal Kbm.

II. 'P. Brodersen 59 Tlemberg Gdm.

Johs. J. Groth 56 Büsum Gdm.

Rud. Thedens 57 Heide Hesteh.

Em. Schlichting 5 9) Kronpr.

Walt. Schlichting 62 $\}$ Kog.

Ch. Stahlschmidt 56 London Magel.

H. Stahlschmidt 56 London Mægl.

F. Stahlschmidt 58 London Mægl.

William Holland 56 London

Arthur Holland 60 London

* P. Petersen Tagkær Gdin.

* P. C. Hansen 57 Tyrstrup Gend.

* Johs. Hansen 58 Tyrstrup Degn

* Jørg. Barsse 62 Skovbøll. ( $\mathrm{G} d \mathrm{dm}$.

* Ernst Asboe:) 63 Grønland Br.M.

* J. Brodersen $\left.{ }^{1}\right) \quad 56$ Llemberg Gdm.

"Forf. til "Fra gamle Dage". \& Miss, is. Afrika 1990-I927.

1872

Søren Ilorlyck 58 Bastrup Gdm. H. Uldall Juhl 58 Binderup Gdm. 


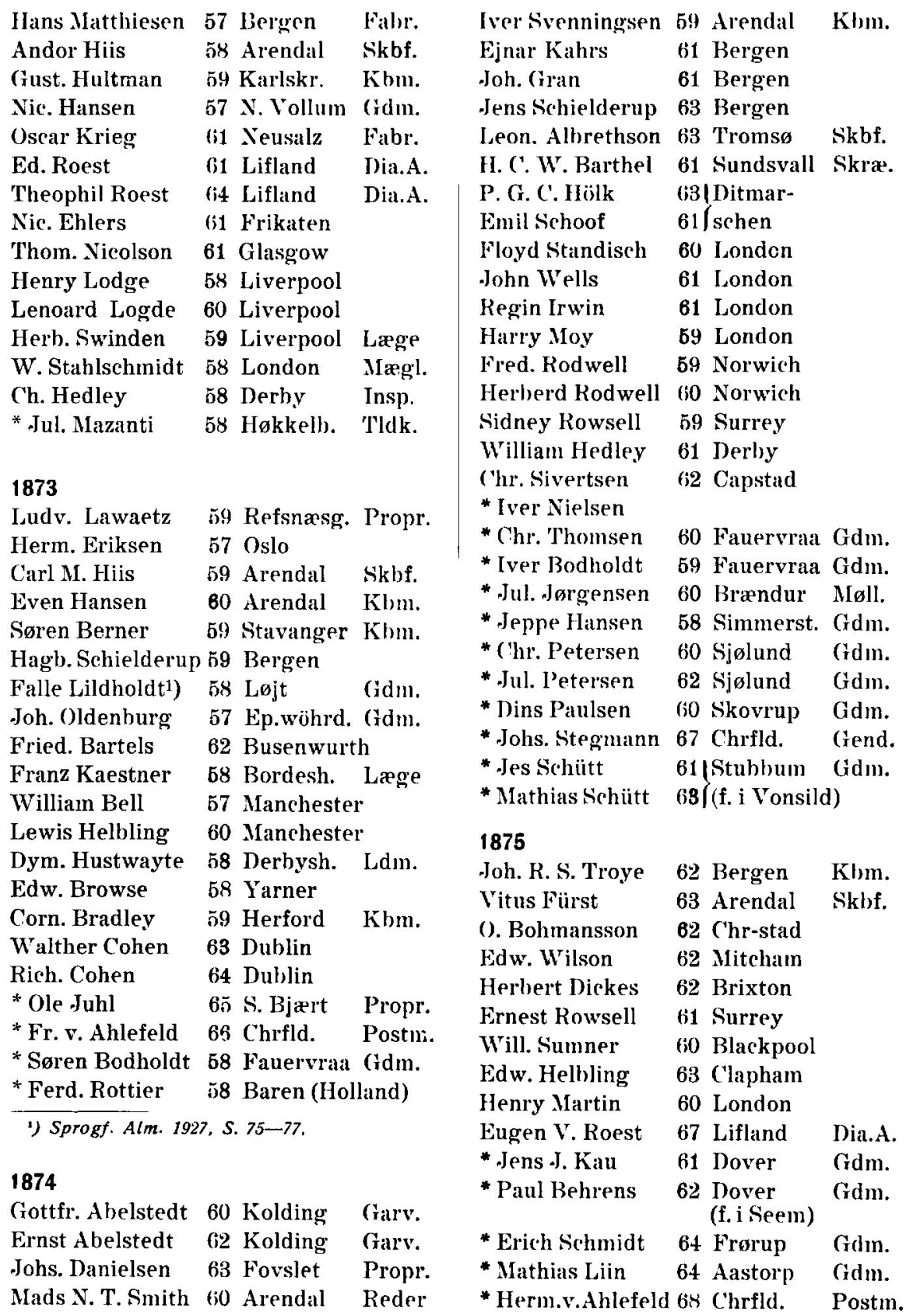


* Niels Schau

* Peter Schau

* Friedr. Asboe
* ()tto lBeck

(i3 Aller

* Iver Matthiesen 61 Tyrstrup

* Jens Kallesen $\quad 65$

${ }^{*}$ Bernh. Albertsen 69

${ }^{*}$ A. Westergaard 61

\section{6}

Hans Hørlyck 63 Bastrup Gdm. Ambrosius Berner 65 Stavanger Khm. Joh. N. Kløcker 62 Arendal Christopher Blick 63 Arendal Sagf. Jørg. Løversen 64 Sandviken Skbf. C. Andersson $\quad 65$ Landskr. $\mathrm{Kbm}$. J. Fr. Mommsen 64/ Kværn- Forp. Sønnich Mommsen 66 holt Forp.

* Andr. Barsøe 68 Skovbøll. Gdm.

*Heinr. Buchholdt 64 Fauervraa Kbm.

* Fr. Buchholdt 66 Fauervraa Khm.

- Aug. Buchholdt 67 Fauervraa Kbm.

* Jac. Jessen 61

- Jul. Ahrenkiel

- Agidius Holm

* Paul Dall

* Jeppe Corydon

*.J. P. Hansen

- Carl Schlünsen

* Corn. Hansen

62 Stubbum

67 Aller

65 Seggel.

64 Tyrstrup

61 Felsted

67 Chrfld.

63 Goldelund

\section{7}

Har. Grønneberg 66 Kbhn.

Peter Schmidt

H. Christiansen

Hans L. Frost

Andr. Cl. Frost

Oscar Kløcker

Henr. Sunne

Andr.T.Mathiesen 6

L. v. Reventlow

Carl Johannesen

Boy Jensen

Adolf Bruhn

Harry Harrison

Frank Bennet

Ed. Stahlschmidt

Arthur Robson

Hans Sivertsen

* H. Th. Dall

* Ad. P. Bryde
62 Hungerbj. Propr.

62 Stenderup Gdm.

62 Stenderup Gdm.

63 Stenderup Gdm.

63 Arendal Sagf.

$63 \emptyset$. Moland Skbf.

Gdm.

68

64

65 Aabenraa Skbf.

60 Manchester

63 Cedars

62 London

63 England

64. Capstad

63 Tyrstrup Gdm.

63 Tyrstrup Gastg.
64 Husum

\section{8}

Carl F. Møller

Hans L. Møller

Oscar Møller

Halfdan Bugge

62 ()slo

64 (Kjølstad

65 Aas)

62 Oslo

Niels Pedersen (it Staubo

Morten Pedersen 65 Staubo

Chr. Andresen $\quad$ 66.Hadersl. Fabr.

William Andersen 65 Hadersl. Just.K.

Hans Knudsen 63 Højer Kbm.

Hans Feddersen 65 Højer Kbm.

Ebbe Sønnichsen 65 Højer Slagt.

Andr. Jessen $\quad 64$ Bylderup Gdm.

H. Stahlschmidt 64 London Mægl.

* Johs. Ahrenkiel 64 Stubbum Gdm.

* Chr. Pedersen 64 Kabdrup Gdm.

*A. Zachariassen 64 Skobøl Teglv.

* Carl Schmidt 69 Frørup Sognf.

* Chr. Schmidt 70 Frørup Sognf.

* Theod. Schmidt 70

* Johs. Hansen 70 Chrfld. Mejer.

W. Schwarzrock 71 Chrfld. Gend.

* Rud. v. Ahlefeld 70 Chrfld. Postm.

* Johs. Hagge 64

* S. M. Paaskesen 64 St. Andst Husm.

\section{9}

Carl Schmidt 66 Hungerbj. Propr. Claus Frost 64 Stenderup Gdm.

Ad. E. Janssen 69 Hadersl. Lage

M. Chr. Mathiesen $65 \mathrm{Højer}$ Forp.

* Nis Dall $\quad 67$ Tyrstrup Gdm.

* Johs. Neiling 65 Chrfld. Læge

* Gust. Wilckens (i8 Bøgeskov Gdm.

* J.M. Pedersen 60) Seggel. Gdm.

* Iver Dall 68 Seggel. Gdm.

* Hans Holm $\quad$ i1 Aller Gdm.

* Jørg. Sievers 71 Høkkelb. Gend.

* Fr. Schwarzrork 72 Chrfld. Gend.

* Jonas Schütt $\quad 67$ Solderup (ræstg. 


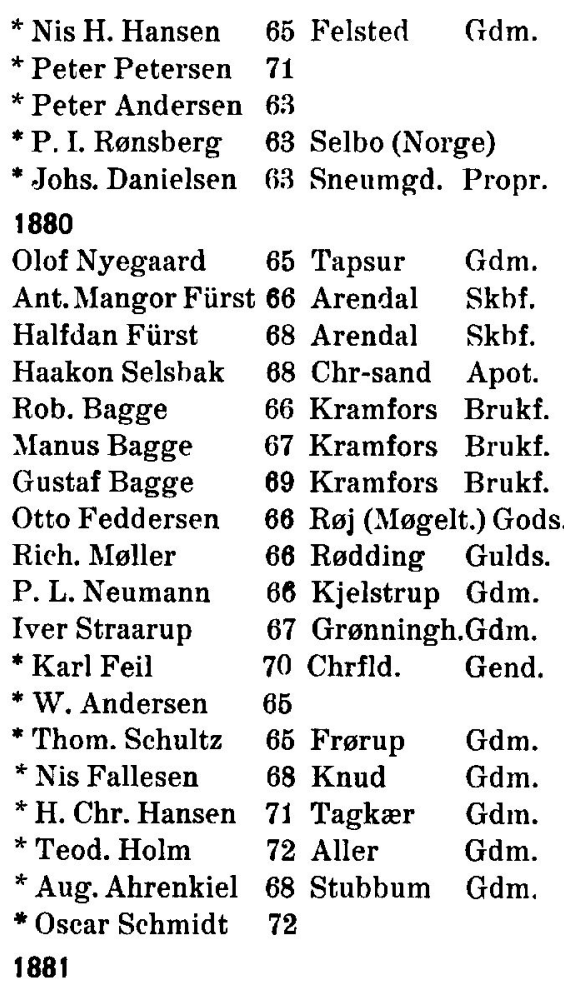

Nic Lorenzen. Andr. Morell Peter Hansen Ad. Cornelsen Heinr. Hansen Falle Kiil Jac. J. Riis Heinr. Lassen * Peter Jessen

* Paul Trummer

" Herm. Wilckens

* Math. Dall

* Hans Dall

* Peter Juhl

* Jes N. Raben

70 Chr-inedal Propr. 68 Oslo Gdm. 66 Wonsmosg.Gdm. 67 Rabsted Gdm. 66 Haderslev

68 Højrup Gdm. 68 Pamhule Gdm. 68 Flensb.

69 Høkkelb. Husm.

66 Frørup Gdm.

70 Bøgeskov Gdm.

69 Seggel. Gdm.

73 Seggel. Gdm.

65 Seggel. Ldm.

68 Fauervraa Gdm.

* Jørg.Chrislensen 65 Fauervraa Ldm.

* Ole Aarup

66 Københ. Gdm.

* MortenSuurballe 68 Lejrskov Gdm.

* Chr. Erichsen 73 Chrfld. Drejer
1882

Sigurd Asp

Wilh. Herrmann

Karl Raben

i0 Hälsingf. Prof.

70 Husum

68 Haderslev Murer

Mareus Neumann 69 Vilstrup Gdm. .Jes Johannsen 68 Vilstrup Gdm.

Fr. v. Buchwaldt 71 Glüksbg. Slotsf.

Rob. Goldstücker 70 Altona Læge

-Johs Dam 74 Labrador Br.M.

* Jørgen Friis 67 Stubbum Gdm.

* Carl Nielsen 68 Agtrup Møll.

* Peter Schütt 68 Hvinderup Gdm.

*P. Refshauge ${ }^{1}$ 69 Hvinderup Gdm.

* Søren Jacobsen 68 øsby Møll.

* Aug.G.Wilckens 70 Kabdrup Gdm.

-Wilh. Wilckens 72 Kabdrup Gdm.

* Hugo Wilckens 73 Kabdrup Gdm.

- Nis Thuesen 67 Aller Gdm.

* C. Hinrichsen 74 Haderslev Tldk.

* Jacob Riis 69 Skærbæk Gdm.

* Nis H. Holm 68 Bodum Gdm.

* Claus Dahl 69

* Walter Padel 75 S. Afrika Br.M.

1) "Bonden, der blev Laudraad“, 1941.

\section{3}

Claës Rudbeck 68 Upsala Oberst

Rasm. Cornelsen 68 Rabsted Gdm.

Hugo Stolley 69 Flensb. Tandl.

Max Kaftan 74 Slesvig Biskop

* Anton Lassen 69 Bramdrup Gdm.

* Rudolf Tams 74 Aller Møll.

* Rasm. Thuesen 71 Gammelby Gdm.

* Knud Raben 71 Fauervraa Gdm.

* Andr. Petersen 69 Bøgeskov Gdm.

* Jac. Becker 74 Aller Præst

* Chr. Boysen 74 Aller

* Mads Schütt 70 Hvinderup Gdm.

* Jørgen Schultz 67 Frørup Gdm.

* Johs Trummer 67 Frørup Gdm.

*Fr. Krüger $\quad 76$ Tyrstrup Gend.

\section{4}

Christen Holst

72 Ringkøb. Just.R. Jac. Jacobsen

71 Oslo Kbm. P. J. Poulsson 70 Trondh. Kbm. 


$\begin{array}{lll}\text { Ernst Nordblad } & 70 \text { Malmø } & \\ \text { Allan Nordblad } & 72 \text { Malmø } & \\ \text { HjalmarTörnquist } 74 \text { Landskr. } & \\ \text { Chr. A. Brodersen } & 71 \text { Slesvig } & \text { Ass. } \\ \text { Hugo Moll } & 68 \text { Hamb. } & \\ \text { Paul de Wolff } & 72 \text { Scotland } & \text { Kbm. } \\ \text { Paul Dam } & 77 \text { Labrador } & \text { Br.M. } \\ \text { * Jes Juhl } & 75 \text { Brabæk } & \text { Gdm. } \\ \text { * Iver Schmidt } & 73 \text { Hoptrup } & \text { Gdm. } \\ \text { * Chr. Kjærbøll } & \mathbf{7 6} \text { Hellevad } & \text { Lærer }\end{array}$

\section{5}

Carl H. A. Ørum

Hans Bang

Johs. Lundbye

Peter K. Petersen 74 Stepping Møll.

Hans Dam

Johs. Knuth

Jul. Stender

Heinr. Topf

Alfred Kreich

Max Kreich

Walter Moltrecht 74 Hamb.

Jan Sluis

Pieter Sluis

* Mart. Hansen

- Barthold Hansen

* Hans Hansen

- Wilh. Jessen

- Nis Thuesen

* Hans Becker

* Nis Bruhn

* Laue Bruhn

* Peter Schmidt

- Chr. Holm

- Erik Wiborg

- Ole Christensen

- Joh. Biester

\section{6}

Hans Pettersen

Chr. H. Winther

Barth. Andresen

Chr. Meyer

Hans Godt

George Sharpley
71 Brændstr. Ldın.

75 Flensb.

74 Slesvig

75 Husum

70 Kiel

74 Kiel

67।Enk-

71/huisen

69|Vonsmosg. Propr.

70 (Vilstrup) Propr.

73 Propr.

76 Brændur Gdm.

72 Fjelstrup Gdm.

76 Aller Præst

72 Hjerndr. Gdm.

74 Hjerndr. Gdm.

71 Sønderbal. Gdm.

77 Rørkær Skbf.

73 Bøgeskov Gdm.

77 Chrfld. Post.

72. Broager

75) Arendal

71 Brede Gdm.

72 Hadersl. Fabr.

72 Kappel Garv.

76 Slesvig

71 Limber
Ed. Nissen

Alf. Brockman

* Erik Work

74 Philadelphia

69 Calcutta

* Johs. Jespersen 72 Hadersl.

Ldm.

* Jes Froin

75 Rørkær

Fabr.

s.skovgd. Gdm.

* Sophus Becker 7o Aller Prast

* Stephan Møller 73 Aastorp Gdm.

\section{7}

Iver Ohlmann 74 Hadersl, Vinh.

Johs. Eckell 74 Vedsted Præst

Johs. Kier 72 Aahenraa Skbf.

Ernst Walter 79 Altona

Eduard Thiele 77 Altona

O. v. Levetzow 74 Pinneb. Postm.

* Chr. Matthiesen 74 Sommerst. Møll.

* Dan I. Petersen 73 Bøgeskov (idm.

* E. Christensen 78 Fallervrila Ldm.

* Jac. Petersen 78 Aller Forp.

* Jesper From 73 Aller Gdm.

* Jos. Hamann 78 Brabak Forp.

* Hans Hamann 79 Brabæk Forp.

* Thomas Petersen 75 Tagkær Gdm.

* Otto Padel 79 S. Afrika Br.M.

\section{8}

Jac. Matthiesen 75 Alslev Gdm.

Jul. Thomsen 73 Billund Gdm.

* Jos. Madsen 80 Cathrinesm.Gdm.

* Jørg. Petersen 80 Aller Gdm.

* Ernst Becker 80 Aller Præst

* J.A.Zachariassen 73 Aller (idm.

* Will. Martensen 74 Stepping (ydm.

* Aug. Dinse 78 Tyrstrup Gend.

* Herm. Klaue 80 Tyrstrup Gend.

* Friedr. König 76 Høkkelb. Toldk.

"Jürg. Lauesgaard 75 Simmerst. Gdm.

* Andr. Lehmann 77 Hadersl. Styrm.

* Knud Dinesen 77 Tagkær Skræ.

* Hans Dinesen 79 Tagkær Skræ.

\section{9}

Andr. Hansen $\quad 74$ Bergen $\mathrm{Kbm}$.

Bengt Wallenius 73 (Göteb.

Adolf Bruhn $\quad 76$ Aabenraa Skbf. 


\begin{tabular}{|c|c|c|c|c|c|}
\hline Jesenius Bruhn & 76 Aabenraa & Skbf. & * Jes Hansen & 78 Tyrstrup & Degn \\
\hline Chr. H. Winther & 75 Laurup & Gdm. & * Andr. Raben & 79 Hvinderup & \\
\hline Adolf.'G. Paetz & 79 Husum & & "Johs. Augustiny & 79 Chrfld. & Postm. \\
\hline Allan Gulliland & 73 Glasgow & & * Will. Suurballe & 77 Chrfld. & \\
\hline James\Robertson & 73 London & & P. Petersen & 82 Chrfld. & Dia.A. \\
\hline Johs. Dam & 74 Labrador & Br.M. & "Oluf_Kragh & 77 Nørbygd. & Propr. \\
\hline Paul Dam & 77 Labrador & Br.M. & ${ }^{*}$ Heinr. Posselt & 77/Gudse- & Møll. \\
\hline * Andr. Juhl & 75 Anslet & Gdm. & * Niels Posselt & Imølle & Møll. \\
\hline Fedder Lund & 81 T'aarning & Mall. & "Siegfried Padel & 78 York & Br.M. \\
\hline Nis Refshauge & 76 Hvinderup & Gdm. & & & \\
\hline P. Frederiksen & 75 Fauervraa & Gdm. & 1891 & & \\
\hline * Erik Fallesen & 75 Skovby & Gdm. & Peter Boyens & 77 Garding & \\
\hline Math. Jørgensen & 75 Skowhy & Gdnı. & Ferd. Tams & 79 Aller & Forp. \\
\hline Johs. Neiling & 81 Chrfld. & Lage & Henning Work & 82 'Tagkær & $\mathrm{Ldm}$. \\
\hline W. Petersen & 81 Chrfld. & Dia.A. & "Chr. Juhl & 77 Anslet & Gdm. \\
\hline Wilfried Padel & S. Afrika & Br.M. & * Jesper Lund & 83 T'aarning & Møll. \\
\hline * Chr.P.Mathiesen & 74 Aarslev & Møll. & $\begin{array}{l}\text { * Niels Møller } \\
\text { * Chr. Jessen }\end{array}$ & $\begin{array}{l}83 \text { Faustrup } \\
77 \text { Vejbøl }\end{array}$ & $\begin{array}{l}\text { Kbm. } \\
\text { Gdm. }\end{array}$ \\
\hline 1890 & & & *Jul. Neiling & 83 Chrfld. & Læge \\
\hline Jac. Neumann & 75 Kjelstrup & Gdm. & Jens Mordhorst & 79 Bøgeskov & Lærer \\
\hline Albert Stolley & 74 Flensh. & 'Tandl. & "Heinr. Scheuher & 83 Tyrstrupg. & Forp. \\
\hline Harold Jones & $7 \tilde{o}$ Brighton & & * Mart. Ohlmann & 77 Hadersl. & Vinh. \\
\hline Luc.des Mesnards & 77 Saintes & & * Laue I. Hansen & 82 Anderupg. & Forv. \\
\hline
\end{tabular}

* Nis Mikkelsen 76 Simmerst. Gdm.

\section{B. Piger:}

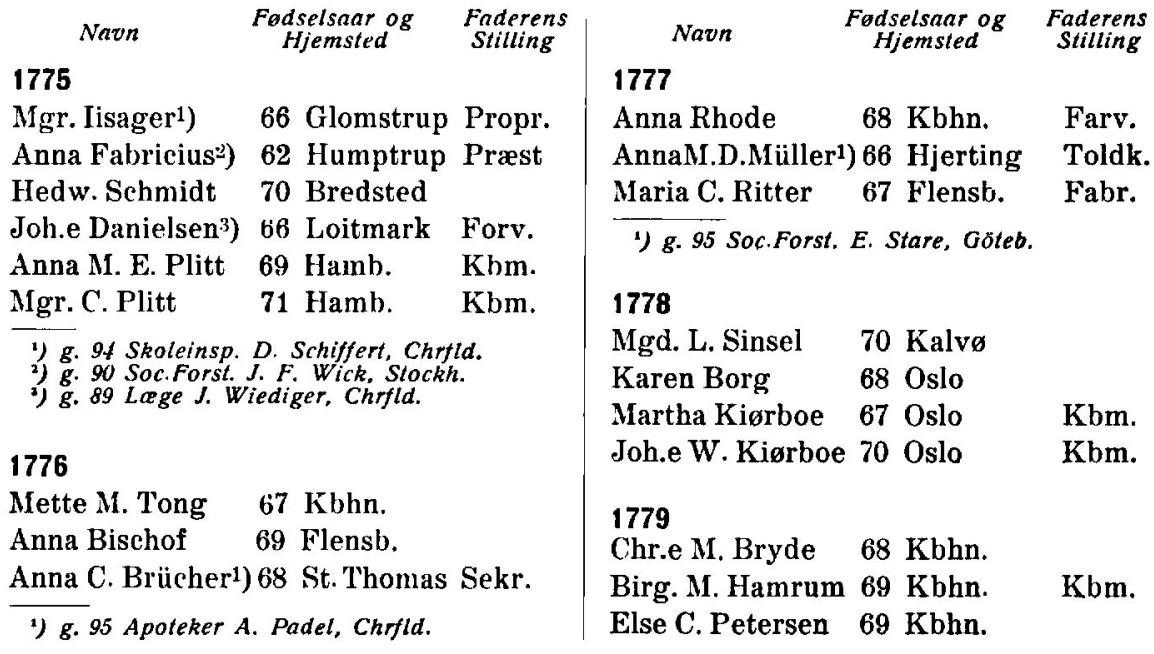


Ingeb. Petersen $71 \mathrm{Kbhn}$.

Abel Hammond $73 \mathrm{Kbhn}$.

Mette Chr.e Abel 74 Stepping Gdm.

Cathr. G. Adolph 71 Nolde Gdm.

Anna B. Petersen 69 Burkal Gdm.

1780

Fr.e Sønnichsen

Chr.e Vothmann

73 Hellevad Møll.

Maria Thomsen

69 Sønderb. Gartn.

71 Ballum Gdm.

1781

Anna B. Lauridsen $70 \mathrm{Kbhn}$.

Else M. Jensen $68 \mathrm{Kbhn}$.

Mette C. Jensen $73 \mathrm{Kbhn}$.

Mgr. Lyngbye 71 Abeltoft Præst

\section{2}

Louise Holm

73 Kbhn.

Gertr. Müller

Mgr. Müller

Anna Allelef

71 Hjerting Toldk.

74 Hjerting

75 Drammen

Ellen Petersen

74 Burkal

Anna S. Bischof 73 Flensb.

Anna Michaelsen 73 St. Croix

\section{3}

Else M. Müller $73 \mathrm{Kbhn}$.

Fr.e J. Schouboe $76 \mathrm{Kbhn}$.

Chre Tang 73 Herning

Chr.e Reiersen 78 Gørding Prorst

Joh.e Santesson 74 Göteb:

Inger Norling1) 71 Göteb.

Anna M. Norling 73 Göteb.

Mgd. D. Norling 75. Göteb.

Maren Geil

78 Tapsur

Anna C. Heinsen 74 Grelsbøl

Maren Thomsen 72 Holm

Anna M. Iversen 78 Lydersh.

Mette M. Adolph') 77 Nolde

Anna H. Petersen 72 Bov

1) g. $92 \mathrm{Kbm}$. A. Kiorboe, Chrfld

2) g. os Gross. J. Hammerich, Kbhn., Moder til Prof. Fr. $H$.

\section{4}

Dor.e Hallander $78 \mathrm{Kbhn}$.

Cathr. S. Buch 79 Kbhn.

Bager

Bager

Bager

Gdm.

Sognf.

Gdm.

Gdm.

Lensf.
Cathr. H. Rhode 73 Kbhn. Farv.

Anna Mgr.Ekman 79 Göteb. Lektor Anna Bonnichsen 79 Bov Gdm.

Maria Thomassen 77 St. Thomas

\section{5}

Cathr.D. Reiersen 80 Gørding Provst

Bodel Gaul 75 Kjærby Gdm.

Cathr. D. Ebbesen 77 S. Bjært Præst

Anna B. Outzen 73 Brede Knipl.

\section{6}

Maria Chr.e Ellert 81 Kbhn.

Dor.e Mgr. Buch 80 Kbhn.

Cathr. M. Røding $80 \mathrm{Kbhn.} \mathrm{Gross.}$

Maren Tang 75 Herning Propr.

Anna E. Bugge 83 Bergen Soc.A.

Mgd. v. Dalman 76 Karlskr. Major

Maria Schmidt 77 Bredsted

* Louise Hegner 79 Barby Br.A.

1787

Joh.e Chr.e v.Barth82 Kbhn. Musik.

Maria Chr.e Stolpe 84 Båstad Dia.A.

Maria H. Suter 75 Ebersdorf Br.A.

1788

Reb.a Chr.e Buch 82 Kbhn.

Mgr. U. Astrup 80 Viborg Stiftspr.

Ingeb. C. Riis 80 Løgumkl. Glarm.

Bot. Thomsen 77 Stokkebro Gdm.

1789

Ulr.e Aarslev 82 Nyborg Kbm.

Fre.e D. Mygind 80 Stepping Præst

Mgr. B. Ritter 84 Flensb. Fabr.

1790

Charl. A. Østrup 80 Vigerslev Præst Anna S. Kiørboe ${ }^{1)} 79$ Oslo Kbm.

Ingeb. Schønfeldt 82 Göteb.

Anna Thomassen 85 St. Thomas

y g. 04 Skoleinsp. S. Sonnichsen, Chrfld. 1791

Gertr. W. Bolte 82 Assens Bager Ing. M. Sjöström 84 Göteb. 
Eva B. Andrén 85 Göteb. Præst Ebba v. Dahlkrona 86 Stockh. AnnaMgd.Bannier 80 Rendsb.

\section{2}

Charl. L. Buch 84 Kbhn.

Soph. M. Clausen 84 Svanninge Præst Cathr. H.Lyngbye 82 Abeltoft Præst Sara Minten²) 85 Göteb Raadm. Volratine Minten 86 Göteb. Raadm. Chr.e Malmström 83 Stockh. Soc.A. Soph. Kibben') 84 Ulvstorp Læge Anna Schmidt 85 Flensb. Kbm.

1) g. a5 Baron C. $H$ o. Posse, Ulvstorp. 2) g. 09 Lage J.J Ekman, Göteborg.

\section{3}

Petron. Aarslev 87 Nyborg Kbm. Ambr. Preuss 86 Kongsb.

Petr. v. Cappelen 85|Drammen Kbm. Anna v. Cappelen 85/Drammen Kbm.

\section{4}

Joh.e E. Minten 83 Göteb. Raadm. M. Elise Minten 86 Göteb. Raadm. Fre.e Car. Minten 87 Göteb. Raadm. Theod.e v. Schröder 85 Tønning

\section{5}

Else Chr.e Andrup 89 Kbhn. Kbm. Mgr. E. Ribben'1) 88 Ulvstorp

M. Henningsen 88 Egernsund Gdm. Anna E Ritter ${ }^{2}$ ) 88 Flensb. Fabr.

1) g. as Hofmarskalk J. A. Lejonmark, Stockh. 2) g. 11 Postm. C. Mygind, Chrfld.

\section{6}

Rosina M. Bath 84 Kbhn Urm. Chr.e S. Thurman 83 Oslo Kbm. Karen Hanson 89 Laurvig Jernv. Anna Valløe 87 Tønsberg Reder Sara Marschall 86 Göteb. Mgd.Gustafschöld 84 Kr-stad Gen.Lt. Maria Petersen 87 Bramdrup Gdm. A.M.Schumacher 80 Nordborg Skom. A. Chre Lind 88 Dynved Gdm. Maria Miecke 91 St.Croix Br.M.

\section{7}

Sara Hülphers 90 Stockh. Gross. BeateChr.eKeyser 91 Stockh. Gross. Sara Chr.e Busch 89 Göteb. Maria Charl.Busch 91 Göteb.

Fre.e v. Bergenstråle ${ }^{1)}$

87 Djurön Baron Anna Kjær Nissen 86 Stepping Gdm. MariaChr.eStrauch88 Zeist Br.A.

* Eva Schönfeldt 89 Göteb.

3) g. 15 Brukspatron C. Tisell, Storberg.

\section{8}

Mette Chr.e Høyer 91 V. Nebel Præst Else M. Bering 88 Skjern Præst Ambr. G. Omsted 94 Tønsb. Cathr. Paulsen 89 Tapsur Maria E. Fink 92 Bredsted Hel. v. d. Smissen 85 Altona Gross. Maria de Jager 87 Altona Gross.

\section{9}

Karen S. Brandt 91 Svendb. Kbm. M. Chr.e Spaak 91 Uddevalla Joh.e Gjærling 92 Göteb. Kbm. Hel. S. Marschall 88 Göteb.

A. Chr.e Windisch 95 Amsterd. Br. Arb. * Elise v. Barth 86 Rendsb.

1800

BeateE.Wahlberg 90 Göteb. Chr.e M. Hansen 91 V. Sottrup Snedk. Am. Schumacher 90 Nordborg Skom. Bened. E. Ritter 92 Flensb. Fabr. Maria E. Sass 96 Hadersl. Apot.

1801

Maria B. Wick 96 Kbhn. Soc.A. Maren B. Andrup $94 \mathrm{Kbhn}$. Kbm. Louise E. Boesen ${ }^{1)} 92$ Vigerslev Provst Anna E. Muhle 92 Erholm Propr. Petrine Muhle 94 Erholm Propr. Ingeb. Mgr. Nilsen 93 Drammen Kbm. Henr.e P. Omsted 95 Tønsberg Agneta de Jager 94 Bergen Gross. Maria Keyser 96 Stockh. Gross. Dor.e M. Hansen 91 Husum Væver 
A.Chr.eBundesen 91 Vellerup Ldm. Helena Linnich 93 Altona $\mathrm{Kbm}$.

? g. 14 Kbm. R. P. Brandt, Nyborg.

\section{2}

(iertr. M. Høyer $96 \mathrm{~V}$. Nehel Prast Hedv. E. Muhle 93 Erholm Propr. Em.a A. Zoëga 94 Stenderupg.Propr. Joh.e Hayken 92 Altona Skbf. Henr.a Hayken 95 Altona Skbf.

\section{3}

If Mod Hapfer 92 Khan Anna Chr.e Møller 96 Svendb. Skom. Maria Lammers 91 Kongsb. Major Edel S. Nilsen 97 I)rammen Kbm. Hedw. B. Stare 98 Güteb. Soc.F. Cathr.Henningsen 94 Egernsund Gdm. A. C. Henningsen 95 Egernsund Gdm. Ingeb.Henningsen 97 Egernsund Gdm. Maria B. Hamel 98 Altona $\mathrm{Kbm}$. Anna D. Steffens 93 Tating

\section{4}

Wilh.e A. Gutfeld 98 Hørsholm Provst Anna H. Gutfeld 90̃ Hørsholm Provst A. Chr.e Crøger 00 Hitterdalen Præst

Joh.e C. Weberg 97 Drammen Khm. Mgd. Weberg 98 Drammen Kbm. Mar.v.d.Lancken 96 Lund Ritm. Sara M. Linnich 96 Altona Kbm. Nan. Hampkens 95 Tating Louise Roentgen 97 Hamb. Klom. Cathr. Heinsen 97 Grelsbøl Gdm.

\section{5}

Joach.eU.Boesen1)95 Vigerslev Provst C'athr.E.Jacobsen 98 Allested Cathr.L Kastberg 94 Stockh. Elisab. Kastberg 98 Stockh. Cathr. M. Todsen 96 Tønder Dor.aWindekilde 96 Vilstrup Prast Maria L. Riegen 96 Altona Cathr. Riegen 98 Altona Mariane Sievers 95 Altona
Maren F. Sievers 96 Altona Kbm. A. Mgr. C.Sievers 00 Altona Kbm. Mgr. Beets 95 Altona Henr.e J. Linnich 98 Altona Kbm.

) g. Pastor S. P. Lassen, Ostrup.

\section{6}

Tabithe s. Plum $95 \mathrm{Kbhn}$ (Stiftspr. Oline Cicilia Plum $97 \mathrm{Kbhn.} \mathrm{JDr.th.}$ Maria Bjørn Muus $97 \mathrm{Kbhn.} \mathrm{Toldk.}$ Anna Nicolaisen'1) 99 Fr-gave SalomeMichaelsen (1) Hugetgd. Propr. Flora Gust.a Stare 01 Göteb. Soc.F. Chr.e Nicolaisen \$6 Løgumkl. Glarm. AnnaD.Nicolaisen 97 Løgumkl. Glarm. Amalie Glöde $97 \mathrm{Hamh}$. Carol. Beckmann 97-Hamb. Joh.eM.Ch.Treü) 97 Finland

1) Underholdes af Greven.

2) dobt Jodinde.

1807

Magna Horrebow 96 Kbhn. Prof. Andrea P.Gutfcld $97 \mathrm{Kbhn}$. Provst Christence Frik 96 Voldberg Møller Maria M. Ernst 97 Slesvig Chr.e Hayken 99 Altona Skbf. Fr.a Horns 99 Breitenb. Hanne Müller 98

\section{8}

Fr.e Chr.e Kall 97 Kbhn. Khm. Maria Soph. Kall 99 Kbhn. Kbm. Soph. Hjort $99 \mathrm{Kbhn}$. Nic.e Hillerup 99 Søbygd Propr. Joh.e R. Jacolssen Allested MariaC.A.Hansen 99 Fr-ia Mette M. Blicher 02 Spentrup Præst Fr.e v. Wistleben 00 Vejle Anna Schmidt 99 Vejle Nic.e Windekilde 98 Fjelstrup Præst Dor.a Harding 02 Elsbüll Lensm. 1809

Cathr. M. 'Tutein $\left.{ }^{1}\right) 00$ Kbhn. Gross. Martha Sorterup2) 98 Næstved Vinh. Elisb. M. Sorterup 00 Nastved Vinh. 
I)or.a ('. Balslev 99 Haarslev Prest Mgr. Chr.e Ploug 99 Faahorg Kbm. ('hr.e C. Ploug 01 Faaborg Khm. Mette Chr.e Tang 99 N.Vosborg Gods. Chr.e Ebbesen 01 Hammelev Præst MetteChr.Paulsen 99 Hajstrup Sognf. (arol.M.Lorenzen 04 Aabenraa Ellen Eskildsen 01 Runde Mølle Joh.e H. Hering 01 Altona Louise Keetmann 98 Hamb. Anna Hayunga 04 St. Thom. Br.M. * Elisab. Adolph 08 St. Kitts Br.M.

) g. 22 C. Frisch, Charlottendal.

3) g. Apoteker C. Moller, Kubenhaun, Vesterbro.

\section{0}

Mgr. (r. Rübner (N) Aalborg Apot. Henr.e Rübner 02)(Hadersl.) Apot. Wedeline Clausen 00 Tønder Husf. Ch. de St. Chenoy 02 llamh. Port.

* Adh.Windekilde 03 Fjelstrup Præst 1811

Herm.e U.v. Kaup 05 Parceel Gods. 1812

Maria E. Gebauer 06 Kbhn. Maler M. C. K. v. Bülow 99 Svendb. Oberst M.S. Hammerich 08 Faaborg Farver Henr.e J. Høyer $03 \mathrm{~V}$. Nehel Præst Mgr. E. Langheim 04 Haderslev Apot. Aug.e W.M.Lassen 99 Slesvig Chr.e Menzel 06 Grønland Br.M. 1813

Arnold.e L. Lund') 02| Gunders- Propr Joh.e Petr. Lund 04Jlevholm Propr. Birg.e Sorterup 03 Nastved Vinh. CicilieS.Sorterup')04 Næestved Vinh. Paul.e Chr.e

v. Rantzau Lehn 03 Hvidkilde Greve Louise A.F.v.Kaup 08|Parceel Gods. Soph. Ad. v. Kaup 09 (Angel) Gods.

1) Moder til Venstrepolitikeren, Overretsprokurator Theod. Leth. ") g. Pastor Hansteen, Samse.

\section{4}

Regina S.Carstens 01 Froskøb. Landf. Maria Mgd. Stare 06 (Gïteb. Soc.F.
Maria Brandt 07 Aabenraa Skbf.

Joh e E. (ijelstrup 07 Skærbæk Præst

\section{5}

Chr.e C. Brandt 03 Svendb. Kbm

Karen (rovinello ${ }^{1}$ ) 08 Nakkebølle

Nina C. v. Holten of Odense Et.R.

Fre.e A v. Holten (5) Odense Et.R.

Maria Rasmussen 06 Bramstrup Propr.

Vill.e Rasmussen 06 Bramstrup Propr.

Joh.e E. Rübner 05 Aalborg Apot.

Anna C. Bau 07 Vanse Dia.A.

Ebba Nordenstedt 06 Stockh.

Anton.e Jansen:) 05 Hadersl.

Nic.e Windekilde 06 Vilstrup I'rast

Wilh.e Hansen 04 Slesvig Kontr.

1) Spansk Soldaterbarn, underholdes af Rosenorn Lehn, Ounebvegd. "U) Underholdes of Amtm. v. Ahlefeld Dehn, Itzehoe.

\section{6}

Ingeb. M. Jepsen 05 Rinkenæs Sekr. Anna Mgr. Tanck 10 Rendsb.

\section{7}

Fre.e C. Gutfeld 05 Kbhn. Provst Henriette Gutfeld Kbhn. Provst

Dora M.Mohr 05 Kbhn.

Nicoline Mohr 07 Kbhn.

Helena Treschow 08 Kbhn. Amtm.

Eline v Bülow 04 Svendb. Oberst

Mgr. Jepsen 03 Rinkenæs Sekr.

Aug.a Block' ${ }^{1}$ ) 08 Glücksb.

Louise Stackmann 08 St. Thomas Br.MI.

Anna Stackmann 10 St. Thomas Br.M.

Maria Stackmann 14 St. Thomas Br.M. 1) Underholdes af Hertuginde Carolina Bruns-
vig-Bevern.

\section{8}

.Joh.e Christensen 05 Khhn. Kbm.

('hr.e M.E.Krogh $09 \mathrm{Kbhn.}$ Brygg.

Ilr.e C. Boesen ${ }^{1)} 07 \mathrm{Kbhn.}$ Gross.

Maria M. Boesen $09 \mathrm{Kbhn}$. Gross.

Nic.e C. Øllgaard $08 \mathrm{Kbhn}$. Kon-

Louise C. (x)llgard $10 \mathrm{Kbhn}$. tor-

Carol.eW. (xllgatard13 Kbhn. chef 
Mariane Schmidt 05 Odense Joh.e Hammerich 12 Faaborg Farv.

リ g. 33 C. F. Balslev, sidst Biskop i Ribe.

\section{9}

Chr.e Nørregaard 11 Kbhn. Kbm. Car.eG.A.Astrup1) 08 Kbhn. Gen.Krigskomm.

Anna Ch.Carstens 08 Erøskøb. Landf. Riborg K. Voigt $\left.{ }^{2}\right) 06$ Faaborg Kbm. Elise Fre.e Voigt 07 Faaborg Kbm. Hansine E. Birch 06 Vedersø Præst Julie E. Birch 07 Vedersø Præst Hedw. Hjorth 10 Flensh.

1) Understottes af Dronningen. $\left.{ }^{2}\right)$ H.C. Andersens forste Karlighed, ifr. $H$. C. Andersens Levmeds bog $1805-31$, udgleet af $H . B$ Bix, 1926, S. 159 ff. $H$. Brix; H. C. A. og hans Eventyr, S. 82-8i (1907): Fr. Bosk: H. C. Andersen, S. $168 \mathrm{ff}$ (1939).

\section{0}

Dor.a Pfefferkorn 07 Kbhn.

Urm. Joh.e D. Gülich $09 \mathrm{Kbhn}$. Mgr. F. Gülich 12 Kbhn. Cathin. Johansen 1) 14 Kbhn. Fred.e Schiødte? 08 Kbhn. Wegul.e Mackholt 11 Slagelse Maria B. Selmer 11 Hørsholm Just.R. Elise M. Lund 09 Gyldenst. Gods. Mar.e L. Døcker 08 Odense Kbm. Louise Th.Døcker 10 Odense Kbm. Jnger Ch. Selmer 11 Vejle Elisab. S. J. Quist 14 Vesløsg. Charl. L. Warnke 14 Stockh. Cathr.M.Thomsen 07 Skodb. Ingeb. Mathiesen 08 Visby Wilh.e Johannsen $08 \mathrm{Fr}$-stad Chr.e M. Graf 15 Surinam Maria Johannsen 13 St. Kitts Soph. Johannsen 16 St. Kitts

\section{Aintm.} Propr. Soc.F. Gdm. Gdm.

Br.M. Br.M. Br.M.

1) Plejedatter af Fabr J. C. Drewsen, Svanemollen. ") Moder til Pastor Johs. Clausen. sidst $i$ Vonsild.

Ca. 1820

$\begin{array}{ll}\text { * Cathr. Paulsen } & \text { Nordsl. } \\ \text { * Chr.e S. Schmidt } & \text { Nordsl. }\end{array}$
* Jac.eWindekilde 13 Vilstrup Præst

* Anne B.Petersen Nordsl.

* Julie Astrup

* Tlrikke Knudsen Samsø?

* Birg. v. Dalman Sverige

* Fred.e v. Dalman Sverige

1821

Vict.e M. T.Ryge1) 10 Kbhn. Skuesp. ClothildeN.S.Ryge16 Kbhn. Skuesp. Anna Beckmann $11 \mathrm{Kbhn}$. Ritm. Simine E. Dons $09 \mathrm{Fr}$-dal Mar.v.Lüttichau 10 Grundet. Gods. Anna R. Wright 14 St. Thomas Br.MI.

1) Skuespillerinde. g. ') 18 Grev Ahlefeldt. " 53 Grev Holck.

\section{2}

Oline S. Rimestad Kbhn. Just.R.1)

Betsy E. Jacohsen Helsingør Bogh.

Laur.e Kroyer Odense

Henr.eJacobsen Aarhus

Isab.a v. Bülow Hadersl.

৩ Birkedommer, Politimester og Vejfiskal.

1823

Car.e M.Petersen 11 Neks $\emptyset$

Henr.e D.Petersen 14 (Fr-stenbrud)

Soph. v. Gersdorf 09 Hanstedgd. Propr.

Maria S. Arndt 12 Glückb.

Elisa Steinthal (1) 15 Hamb. Kbm.

Aug.a Steinthal 16 Hamb. Kbm.

Joh.a F. Lehmann 16 Grønland Br.M.

Anna B. Lehmann 18 Grønland Br.M.

yoble Jodinder.

1824

Henr.e v. Schubart Odense Baron Fred.e Lange $12 \emptyset$ Ørbaklund Lt. Anna Friborg 14 St. Thom. Br.M. Chr.ane S. Friborg 16 St. Thom. Br.M.

\section{5}

Cathr. P. Thune 17 Paaby Præst Amalie J. Valeur 16 Viborg Apot. 
S. R. v. Mansbach 16 Oslo

E.B.D.v.Mansbach18 Oslo
1) Adj. hos Kongen. Chef for den Kgl. Norske Krigsskole.

\section{6}

Julie Hoffmann Chr-sund

Louise C. Schultz 16 Drammen

\section{7}

(am. Wergeland') 13 Eidsvold

Maria E. Herre 17 Oslo Gross.

Joh.a C. Herre 18 Oslo Gross.

Em.e K. Roll 17 Trondh. Ass.

Nic.e C. Jusness 16 Lillesand

Aug.a v. Höpken 13 Stockh. Baron

('hr.ane v.Schultz1) 12 Aller Kpt.

Mathilde v. Schultz 15 Aller Kpt.

Elisa v. Schultz $\quad{ }_{17} I_{\text {Aller }} \quad \mathrm{Kpt}$.

Anna Thygesen 15 Varmark Gdm.

Chr.e Marsch 12 Skast Gæastg.

('hr.e M. Mleyer 17 Ullerup Præst

Aug.a F. Franzén Sørup Præst

Thom.e J. Mun- 17 Langen- Krigs-R. derloh felde

1) g. Grosserer Collet, Forfatterinde, Skildring af Skoleopholdet i Chrfld. $i_{n}$ I de lange Natter". J893, heri ogsaa Skildringaf Veninden Christiane, som dode lige efter Konfirmationen

\section{8}

Maria C. Meinert 21 Kbhn. Mgr. Lindstedt 19 Lerbæk Forp. ('arol.C. Lindstedt 23 Lerbæk Forp. Amalie Kjøsterud Drammen Betsy Eckersberg 20 Drammen Joh.a B. Johnsen 12 Oslo Adolf.e M. Smith 15 Oslo Thom.e Jusness Ll.sand Carol. Lorenzen 15 Svanstrup Gdm.

\section{9}

Louise Solem Oslo Elisab. Grøndahl Oslo Bogtr. Joh.a H. Moe $16 \mathrm{Chr}$-sund Lt. Chr.e A. Moe $17 \mathrm{Chr}$-sund Lt. Carol. Thomsen 16 Skodborg Sognf.

Glarm.

O-sagf.
Charl. v. Laer

Beierholm Propr. Anna B. Nielsen 16 Brede Gdm.

1830

Charl. C. Schow 18 Oslo

Mart.e Howland 17 Oslo

Agnes L. Grïning 16|Ondals Konsul

Jeanette Grüning 17/Jernv. Konsul

Beate S. Warnke 21 Stockh. Soc.A.

\section{1}

Cathr.v.Stockfleth Kolding

1832

Soph. Knudsen Samsø Insp.

Joh.a M. Kjær 14 Rømø

Henr. Isernhagen 17 Altona

1833

Maren N. Müller 21 Aastorp Ldm.

1834

Joh.a S. v. Posse 19 Ulvstorp Baron Bolette Thomsen') 21 Skodborg Sognf. Chr.e C. Thomsen 22 Skodborg Sognf.

ग) g. A;H. A. Kritger, Bevtoft.

1835

Chr.e Tranberg 24 Varde Kbm. Petrea Cohn 20 Varde Kbm.

Bred.e Riddervold Fr-hald Provst

Hanna Lund Fr-hald

Kaja Boysen Fr-hald

Clara Bager Malmø Kbm.

Beate Tøgesen 23 Stenderup Ldm.

Carol.Ihrer Grønland Br.M.

1836

Nielsine Knudsen 22 Samsø Insp.

Emma Knudsen 23 Samsø Insp.

Caroline Lund 30 Skjern Dia.A.

Petron. Seglun 20

Hedv, v. Fröhlich 19 Katrineb. Baron

Anna Thomsen 24 Skodborg Sognf.

Joh.e Mehlhose 28 Grønland Br.M.

Mary Münzer 28 Antigoa Br.M.

*Helene Ritter 26 Flensb. Ldm. 
1837

Amalie Cohn

Chr.e Petersen

Maria Müller

Joh.e Michaelsen

Hildur Scheving

Julie A. Hansen

Henr.e L. Pfeiffer

* Emma Bock

* Chr.e Bock

\section{8}

Marie Leth

Chr.e Lassen

Laura Dah!

Amalie Friis

Ingeb. Holgersen 22 Hadersl.

Jensine Holgersen 20 Hadersl.

Claudine Mammen Fr-stad

Aug.e Mammen

Laur.e C'antor

Fr-stad

Mgr. Pimberton 28 St. Croix

Esther Pimberton 31 St. Croix

Henr.e Kintling

\section{9}

Rud.e S. Lund 33 Skjern

S. Quisl. Sandberg 24 Fr-stad

Olafva Bèen 26 Hälsingb.

Charlotte Bèen 27 Hälsingb.

Marie C. Petersen 25 Aastorp

Math.e Jørgensen 26 Brændur

Soph. Davidsen 24 Aabenrala

Anne M. Petersen $25 \mathrm{~V}$. Alslev

* Laur.e Hansen 33 Skanderb. Murer

\section{0}

Anne S. J. Jermiin 27 Aasumgd. Propr.

Mette Hansen

30 Eltang

Mariane C. Blom

Mariane Ottesen

29 Drammen Skbf.

Chr.e M. Melsom 25 Tønsherg

Cathr. Bugge . 22 Oslo Rektor

Chr.e van Laer $2 \overline{5}$ Beierholm Propr.

Nic.e Matthiesen 26 Oversø Præst

*Aug.e Salling 28 Vonsild Præst

\section{1}

Elise v. Smidten 28 Faaborg Herrf. Louise Ilusted 29 Ringkøl). Kbm. ('hr.e Munksgaard 23 Ringkob. Kbm. Marie Riddervold $27 \mathrm{Fr}$-hald Provst BoelM.Riddervold $30 \mathrm{Fr}$-hald Provst Anna M. Faye 29 Fr-hald Kbm. Wilh.e Th. Lund $29 \mathrm{Fr}$-hald Kbm. Betsy (. Neyer 26 Fr-værn Kpt. Thura L. Gude 29 Rygge Præst Mgr. Petersen 28 Dally Propr. Soph. E. Matzen 28 Torning Gdm. Carol. Renkewitz 35 Jamaira Br.M.

* Elise Page 24 London

* Emma Münzer 35 Antigoa Br.M.

\section{2}

Isab.v.Benningsen 32 Nastved Ritm. Anna M. Moller 33 C'onst-borg Propr. Chr.e H. Lassen $27 \mathrm{Fr}$-stad Lt. KajaA.Diedrichson28 Fr-stad Kpt. .Just.e W. Bing $30 \mathrm{Fr}$-stad Sorskr. (ijertinde Valløe 28 Tønsberg Reder Marie Wright 30 Langesund Prast Cathr. Hauff 24 Sandefj. $\mathrm{K}$ hm. Kathinka Garman Fr-hald C'lara Voigt 28 Oslo St.R. Eline Kjær 29 Straarup (Idm. Anna B. Winther 29 Abterp (Gdm. Mgd. Mathiesen 28 Højer (idm.

*Julie Holtermann Trondh. Prest.

\section{3}

Laura Hygum 28 Kbhn. Postk. ('hr.e M. Koefoed 33 'Timgd. Propr. Laur.e M. Holbeck 32 Albak ('athr. Hvistendahl 30 Tonsh. Kbm. Annet.Hvistendahl30 Tønsb. Kbm. Marie Ingier 29 Skien Præst Susanne Hauff 31 Sandefj. Kbm. Inger Jacolssen $29 \mathrm{Fr}$-værn Ellen D. Arup 30 Drammen Biskop Cathr. C.Arup 30 Drammen Præst Anna (. Collet 32 Drammen $\mathrm{Kbm}$. Wilh.e L. Collet 34 Drammen Kbm. Eline C. Dahl 32 Irammen Byf. 
Hedv. M. Rasch 32 Drammen Sorskr. Karen Mgd. Bugge 29 Stordalen Prorst Maren J. Bugge 32 Stordalen Provst (athr.Mgd.Flood 31 Trondh. Præst Marie M. Flood 33 Trondh. Præst Ebba Follin 30 Hälsingh. Ritm. Marie C. Tugesen 29 Stenderup Ldm. Else Frey 29 Galsted Gdm. Henr.e D. Fries 30 Illerup Forp. (Ballegd)

Maria A.Wolf. 30 St. Croix Br.M. * Anna Sønnichsen 32 Ladelund Ldm.

* Julie Page 28 London

* Cathr. Page 30 London

\section{4}

Adam.e D.M.Lund 38 Skjern Amalie C. Lïttken 27 Ringkob.

Anna M. Jensen 24 Ringkob. Farver Sophie Schiotz 32 stavanger Byf. Oline Svendsen 29 Stavanger Kbm. Bojine Børresen 30 Stavanger $\begin{array}{lcc}\text { Abigael Dircks } & 31 \text { Aamodt } & \text { Præst } \\ \text { Dor.a Barsøe } & \text { Moss } & \end{array}$

Dora Petersen Moss Konsul Bolette Nissen (1) Oiers Provst Nicoline Nissen $\}^{1}$ ) Oiers Provst Alida M. Clausen $30 \mathrm{Chr}$-sund Konsul Henr.e Kjempe 31 Chr-sund Skbf. Carol. Nicolaisen 29 Løgumkl. Glarm. Aura M. Lund 38 Surinam Br.M.

ग) Niècer til Oberst Fibiger, Kbhn.

\section{5}

Anna Frick 31 Rosenlund Gods. Marie M. Frick 33 Rosenlund Gods. Anne E. Hagerup 30 Trondh. Kpt.It. ('aspara Hagerup 32 Trondh. Kipt.lt. Nic.e L. Groent ved 82 Oslo (hristopha Budde 33 Porsgr. Fre.e Ch. Ingier 81 Romnæs Hanna E. Ingier 33 Romnæs EliseWestergaard 29 Arendal Carol. Aagaard 33 Hlognæs Helena Croger 29 stavanger Juliane Petersen 30 Dalby Præst Kbm. Propr. Kpt. Propr.
* Mlath.e Olsen

* Gustava Olsen

* Petra Biorn

* Chr.e Johansen

1846

Fanny Zeuthen $30 \mathrm{Kbhn}$. Kbm. ('arol. L. Zeuthen $32 \mathrm{Kbhn}$. Kbm. Math.e Finne 29 Oslo Laura Kock 32 Oslo Thura Oppergaard 30 Oslo Randine Holm 30 Buskerud Betsy Hutchinson 32 Drammen Kbm. Lucie Ch. R. Mohn 34 Bergen Kbm. (iottliebe Mohn Bergen Kbm. AnnaM.Humeland 33 Stavanger Skbf. Jacobine Toft $\mathbf{3 5}$ Arendal $\mathrm{Kbm}$. Joh.e B. Toft $\quad 36$ Arendal Kbm. Anna Björkman 33 Malmø Khm. Fre.e Mathiesen 31 Højer (rdm. * Anna Thraen 37 Antigoa Br.M.

\section{7}

Nic.e M.N.Røding $34 \mathrm{~K}$ Jhn. Gross. Ida W. Schiander 32 Moss Fuldm. Sine M. Nickelsen 32 Oslo Joh.e M. Struck 36 Oslo Elise F. Clausen $33 \mathrm{Chr}$-sund Konsul Mariane C. Clausen $35 \mathrm{Chr}$-sund Konsul Helene M. Dahl 35 Fr-værn Toldk. Henr.e S.Paus 32 Elverum Sagf. Anna Sjökrona 32|SkönoPauline Sjökrona 34/bæk Mar.e Björkman 38 Malmø Kbm. Emma M. Dahl 38 Göteb. Mægl. Fanny Lehmann 33 Göteb. Gross. Cathr. Petersen 32 Dalby Propr. Doris S. Petersen 32 Hoptrup Præst Sorskr. Minna S. Petersen 34 Hoptrup Præst Tldass. Wilh.e Schmidt 3õ Berlin Præst *HeleneF.Thraen 39 Antigoa
* Ingeb. Nissen 38 Burkal

Br.M.

Gdm.

* Anna Nissen 33 Burkal Gdm.

* Chr.e Bruhn 31 Trondh. Kpt.

*Inger Christensen 40 Øsløs Gdm.

* Aug.a Ritter 32 Flensb, Ldm. 
1848

Cathr. Boesen 35 Vejle Kbm.

Hanna Berg Moss

Mathie Petersen 36 Hadersl. Sadel.

Cathr.Christiansen 34 Siverkro Gæstg.

* Marie Engström 33 Drammen

* Carol. Paulsen 32 Uberg Gdm.

\section{9}

Joh.e K. Knudsen 35 Samsø?

Cathr. C. Scheel 35 Oslo

Helene M. Scheel 35 Oslo

* Charlotte Rolfs Nordsl.?

* Anna Daugaard 34 Grejs Snedk.

* Dor.a Hansen 33 L. Barsbøl Propr.

* Charl. Buchwaldt 35 Slesvig Ldm.

* Emil. Buchwaldt Slesvig Ldm.

\section{0}

KajaS.Gundersen 38 Stavanger Kbm.

Chr.e Fasting 36 Levanger Toldk.

Marie Tonnesen 37 Stenderup Gdm.

Chr.e M. Frost 36 Stenderup Gdm.

Emma Petersen 34 Hadersl. Postm.

Laurentine Fehr 40 Stepping Præst

* Bertine Andersen 44 Tyrstrup Forp.

\section{1}

Maren E. Malling 36 Oslo

Caroline Lund 37 Farsund

Hilda Österberg $40 \mathrm{Chr}$-stad

Joh.e E. Hansen

Anna H. Kall

35 Hadersl.

Marie Nissen

35 Ejsbøl

36 Havsted

Dor.a M. C. Otte 45 London

* M. Volquardsen 35 Hadersl.

* Bodil Jessing $35 \emptyset$ Ørsted

* Martine Lund

42 Grønland

\section{2}

Car.F.Lindegaard 40 Svendb. Math.e Petersen 42 Odense

Dor.a L. Glitsch 41 Middelf.

A tte $B$.Johnson

Math.e N. Lind

39 Trondh.

Louise M. Tams
Bogtr.

Skbf.

Bygm.

Apot.

Gdm.

Gdm.

Skom.

Toldk.

Ldm.

Br.M.

Skom.

Farver

Glarm.

Gdm.

Møll.
Adam.e Feddersen 38 Mogelt. GodsCarol. Feddersen 40 Møgelt. \}insp. Marie D. Grauer 38 Kliplev Præst Anna L. Lund 43 Grønland Br.M. Berta Schulz 44 Ndietend Br.A.

\section{3}

Anna E. Holders $42 \mathrm{Kbhn}$. Guld. Ida A. Holders $45 \mathrm{Kbhn}$. Guld. Hildur Jacobsen 38 Uslo Toldk. Cæcilie Meyer 39 Skodborg Gdm. Marie C. Winther 39) Brede Knipl. * Anne M. Nielsen 40 Harres Gdm.

* Henr.e W. Dörr 43 Jægerspris

* Henr.e Bertram 40 Cathrinel.

* Eleonore Ritter 38 Tyrstrup Gdı. 1854

Agnes Holst 44 Frørup Gdm. Ing Finnemann 40 Taarning Gdm. Cathr. Mgd. Skøtt 40 Seggel. Gdm. Marie C. Wiborg 40 Bøgeskov Gdm. Anna C. Jensen 39 Vedsted Præst Maren Hansen $\mathbf{4 0}$ Hjolderup Gdm. (arol.H.Winding 41 Højer Lage ()ttilie Todsen 39 Hojer (idm. Andrea Hinrichsen 41 Højer Kbm. Herle Nicolaisen 41 Mølby Mall. Lucie Jensen 43 Trausted Gdm. Anna Brodersen 39 Lydersh. Gdm. Aug.a Meynell 46 Dublin

1855

Thora Repsdorph 41 Holstebro Sagf. Elise C. Wittrup 42 Mariager Præst Dor.a C. Tønsberg 40 Oslo Konsul Marie M. Bruhn 41 Aabenraa Skbf. Anna Jørgensen 39 Hadersl. Garv. Anna M. Ravn 41 Tyrstrup Gdm. Betty Ch. Holst 40 Frørup Gdm. Camilla Petersen 46 Hjerndrup Gdm. Carol. F. Tams 41 Aller Mroll. Ing. L. M. Holm 41 Aller Gdm. Soph. M.Lorenzen 41 Knud Gresty. Mgr.M.Lorenzen 42 Knud (iæstg. Dor.a Simonsen 42 Gjesten Gdm. Emilie Sønnichsen 40 Hestholm Gdm. 
1856

Joh.e C. Ferslev

Marie H. Berg

Charl. Krarup

Elise Clausen

Hanna Clausen

Marie Marstrand

Ida H. Marstrand

Reg.e Rognskoug

Carol M. Salicath

Laura Isaachsen

Math.e Lâsthom

Ing. Berthelsen

Dor.a Højsager

Marie F. Uldall

Mariane Sørensen 44

Mgr. Ravn

Eline N. Jacobsen

Cathr. L. Lund

Wilh.e S. Obel

Laura H. Ganner

Jens.e Neergaard

Meta Feddersen

Anna Nissen

Annette Todsen

Mgd. Mommsen

Marie Brodersen

Goda Lorenzen

* Chr.e Staal

* Jensine Staal

* Gustava Staal

\section{7}

Aug.e Rasmussen 43 Samsø

Fre.e Hinselmann 42|Lerbak

Elisab.Hinselmann 43 (Mølle)

Chr.e Ganner

Bertha Simonsen

Joh.e Martens

Henr.e Petersen

Elsbe Hansen

Anna C. Jespersen

Chr.e Nissen

Anna M. Tanck

43 Hadersl. Slagt.

45 Hvinderup Gdm.

45 Drenderup Propr.

46 Drenderup

42 Hjolderup Gdm.

Carol. Feddersen 43 Røj
Lydia Hatt

43 Zeist

Anna Schwensen 50 Surinam

Maria Schwensen 52 Surinam

*A.Christophersen 42 Oslo

Br.M.

Br.M.

Vognm.

\section{8}

Elisab. Sabroe 44 Alstedgd. Propr.

Mgr. Fabricius 46|Bram- Propr.

Term.a Fabricius 46/mingegd. Propr.

Nina Haurowitz 49 Aarhus Skuesp.

Agnes S. Frey 46 Oslo Hattem.

Kathinka M. Frey 48 Oslo Hattem.

Soph. A. Tønsberg 45 Oslo Konsul

Thalet.Gundersen 45 Trondh. Skræ.

Mally V. Johnsen 45 'Trondh. Glarm.

Joh.e Jørgensen $43 \mathrm{Chr}$-sund Apot.

Lydia Dahl 45 Chr-sund Kbm.

Doris Clausen $44 \mathrm{Chr}$-sund $\mathrm{Kbm}$.

Amanda Bagge 47 Kramfors Forv.

Thekla Fogelklou 46 Malmø Kass.

Elisab. Feddersen 45 Møgelt. Insp.

Caecilie Todsen 42 Tønder Kbm.

Harriet Slater 38 London

*Gunde Lind 43 Hadersl.

1859

Cathr. M. Wolff 44 Herningh. Propl.

Paul.e Hinselmann 45 Lerbæk Forp.

Helene Schmidt 45 Hoptrup Gdm.

Chr.e E. Kragh 46 Hoptrup Gdm.

Cecilie Kjær 45 Hadersl. Maler

Anna Seneberg 45 Hadersl. Bogtr.

Cathr. E. Beyer 44 Skærbæk Gdm.

Marie Brodersen 45 Hojer Insp.

Joh.e Feddersen 1 ) 46 Møgelt. Insp.

Louise Koopmann Altona

Elise Jannasch 51 S. Afrika Br.M.

J) g. $\$ 1$ Fr. Hayberg, Visby.

1860

Carol. Clausen $46 \mathrm{Chr}$-sund Konsul

Harriet Bennecke 46 (Oslo

Anna Siems 45 Ørkedal Propr.

Ida Björkman Malmø Kbm.

Hansine IIdall 45 S. Bjært Gdm.

Anna C. Schultz 47 Dalby Gidm. 
Bothilde Beyer 46 skxrbak (idm. ${ }^{*}$ Mgd. Have 49 Frorup Gdm.

Marie Grauer 46 Rise Præst * Botille Ilave 52 Frorup Gdm.

*Anna Simonsen 48 Gjesten Gdm. * ('hr.e Finnemann 50 'Tatarning Kbm.

* Maria Berg 46 Aalhorg Klom. *.Joh.e Brandt 48 Bjerndrup Væver

* Maria Schønberg 44 Svodborg(?)Bager 1864

1861

Joh.a W. Ferslev

Maria Ferslev

Anna Lokke

Emma Lokke

Henr.e Vibe

Alice Bock

Soph. Österberg

A.Chr.e Petersen $\left.{ }^{1}\right)$

Cathr. Lauritzen

Doris Lassen

48 Horsens

50 Horsens

49 Trondh.

48 Oslo

49 Oslo

$48 \mathrm{Chr}$-sand

46 Chr-stad

Aug.e F. Herbrich 53 Gronland

Adolf.e Herbrich 5ă Grønland Ingeb. Asboe

53 Gromland

* Chr.e Müuller

50 Hadersl.

* Louise Gron

47 Fr-hald

* Sylvia Jacobsen

7 Lang $\emptyset$

(Oslo)

'Jiel. Pastor Scheel, Abild, Moder til Prof. Scheel.
Kith

1862

Nanna C. Engh 49 Raavold Sorskr.

Maria Wischmann 53 Oslo

Hort.e Ringheim Karlskrona

Maria Kylling 51 Bogeskov Gidm.

Dor.a Friedrichsen $47 \mathrm{~V}$. Terp Gdm.

Elise Petersen 47 Hojer Kbm.

Hanne E. Lübbes 47 Egernf. Sagf.

* Emma Juhl

* Cille Sørensen

Chrfld.

* Luise Larsen

4!) Fovslet

47 Mandal

1863

Emma C. Ferslev 62 Horsens Anna D. Magnus 49 Oslo

Hanna Smith 52 Skeberg

Leonore C'lodius 52 Tromsø

Marie Kullmann 50 Chr-stad

Ingeb. Brodersen 48 Lydersh. (idm.

Bot.e.M.Brodersen 48 Ulemberg Gdm.

\section{4}

Louise Roscher 51 Ringerike Dir.

Marie Buchholdt 52 Langkar Gdm.

Chr.e Dethlefsen 51 Rørkær Gdm.

Marie Asboe 57 Gronland Br.M.

* Meta Schmidt 56 Fauer-

vraagaard Forv.

* Ida Juhl 56 Chrfld. Lage

* Anna v. FischerBenzon 49 Chrfld. Toldk.

* (Thr e v. Fischer-

Benzon 52 ('hrfld. Toldk.

* Elise v. Ahlefeld 60 ('hrfld. Postm.

\section{5}

Soph.v.Arenstorff is ()vergd. Propr. K. K. v. Arenstorff 55 Overgd. Propr. Math.e Roed 50 Eriksholm Forp. Anna ('. Engel 57 Aarhus Bager Cathr. Dietrichson 53 Drammen Sorskr. Emma L. Holst 52 Ronhojgd. Forp. Soph. Valentiner 56 Tyrstrup Prest *Agnes Lehmann 54 Tyrstrup Slagt.

* Mgr. Autzen 52 Tyrstrup Gdm.

* Maria Schmidt 58 Fauervraa Forv.

* Anna K. Hansen 51 Andrupgd. Forv.

\section{6}

Anna v. Holstein 5: Eriksholm Kam.h. Kirstine B. Friis 53 Stubbum Gdm. Soph. (Hrauer 53 Burkal Prast

* Anna v. Ahlefeld 56 chrfld. Postm.

\section{7}

Propr. Anna Albrethson 55 Tromsø Skibsf.

Kbm. (iertha Dahl 54 Skeberg Lensm.

Ing. K. Kahrs 53 surendalen Læge

Khm. Thyrese Bruhn 53 Bramdrup Gdm.

Ing. Brodersen 52 tremberg Gdm.

Hel.e Lichtfeld 53 Ammersw. Gods.

Elisab. Schmidt 55 Meldorf. Vinh. 
* Anna Bogh

¿õ Binderup) (idm.

('hr.e ()mholt

58 Arendal

Khm.

* Mette Lind 52 S. Bjart (idm.

* Amalie Schmidt G1 Fauervara Forv.

\section{8}

Anna Wolter

59 Kbhn.

Soc.s.

('ara J. Petersen 1 ) 5o Dalby

Propr.

Marie Hegge

57 Trondh.

Hattem.

Cam.aChristiansen 5s Trondh.

(rastg.

Chr.e ()hlsen

53 Hadersl. Skom.

Anna M. Dahl

58 Taarning (idm.

Adele Meyer

56 Siesebye Læage

* Anna Asboe

59 Grønland Br.M.

* Maria Barsoe

54 Skovbgll. (idm.

* ('hr.e Barsoe

59 Skovboll. (idm.

1) g. Pastor Doose. Stepping.

\section{9}

Marie Jorgensen 04 Stenderup ( $\mathrm{x} d \mathrm{~m}$.

Chr.e Jorgensen 54 Stenderup ( $\mathrm{xdm}$.

Mgr. Hørlyck 57 Bastrup Gim.

Marie Michaelsen 57 Ladegd. (idm.

Joh.e Lildholdt 56 Løjt Gim.

Meta Boesen 5̄ Mjolden Gidm.

Am. Matthiesen 55 Randerup Gdm.

Carol. Toft 56 6 . Terp Gdm.

Carol. Lück 55 Meldorf Reder

Hel.e Schlichting 56 Kronpr. Kog (idm.

Emma Thedens 56 BusenwurthHesteh.

Wiebke Lichtfeld 56 Ammers- Gods. wurth

* Emilie Asboe

61 Grønland Br.M.

* E. Ferdinand

(3) Chrfld. Toldk.

${ }^{*}$ Lisette Bolte 55

\section{0}

Carol. Hørlyck

Carol. Frey

56 Bastrup Gdm.

57 Oslo Hattem.

Cathr. Schlichting 57 Kronp. Kog Gdm.

* Sophie Larsen (i2 Jamaica Br.M.

* Louise Guericke Grønland Br.M.

* Carol. Asboe

* Louise Bock

6. Gronland Br.M.

62 Bobol Sgnf.

\section{1}

Karen Omholt 53 Arendal Kbm.

Hanna Th. Omholt 55 Arendal Kbm.

Ing. (inndersen 50 Arendal Khm.

Amalie Gundersen 60 Arendal Kbm.

Anna Hammer 58 Arendal Konsul

Anna Thede 56 Hadersl. Hesteh.

Marie s. Adolph 57 Nolde Gdm.

Cathr. Ballein 62 s. Afrika Br.M.

Kaja Ballein 67 S. Afrika Br.M.

** Maren Petersen 57 Hammelev Gdm.

* Emilie Ravn 57 Kjelstrup Gdm.

* Chr.ce Petersen 58 Tapsur Gim

* Dor.a Petersen 59 Tapsur Gdm.

* Carol. Schmidt 63 Fauervraa Farv.

\section{2}

Adele Andersen 59 Haugesund

Soph. Mathiesen 60 Bergen Fabr.

Anna Shetnan to Chr-sund Skibsf.

MaryStahlschmidt58 London Mægl.

Ed. Stahlschmidt 60 London Mægl.

* Chr.e Beck 6.2 Taarning Møller

* Anna Dahl 58 Tyrstrup

* Anna Andersen 57 Fanervraa Gdm.

* Dor.a Schröder 64 Tyrstrup Kontr.

* Adolf.eSchröder 65 Tyrstrup Kontr.

* Mette K. Sabro 58 Tarm Insp.

* Math.e Asboe 65 Grønland Br.M.

\section{3}

Dor.a Hørlyck 60 Bastrup Gdm.

Joh.e C. Hansen 57 Arendal Kbm.

Marie Hansen 59 Arendal Kbm.

Helene Gjertsen 59 Bergen Kbm.

Anna Gjertsen 62 Bergen $\mathrm{Kbm}$.

Bertha (ioll 61 Bergen Kbm.

Mgr. M. Moller 61 Oslo Kbm.

Hilda Koch 64 Bodø Konsul

Anna Nissen 59 Havsted Gidm.

Elise Göttig 63 Flensh. Propr.

Aug.a Frenssen 1) 57 Barlt Snedk.

* Marie Bargum 60 Høxholt Gdm.

* Chr.e Hansen 59 Hoxholt Gdm.

* Kirsten Sørensen $59 \mathrm{~V}$. Alslev Gdm.

- Cath. Sonnichsen60 Tyrstrup Ldm.

1) Saster til Digteren Gustav Frenssen. 
1874

Angla Svensson 60 Kolding (iæestg. Cam. Wischmann 65 Oslo Fotogr.

Emilie J. Wohl 60 TelemarkenRitm.

- MarthaHoffmann 62 Tyrstrup Brygg.

* Kunig. Stegmann 64 Chrfld. Gend.

* Anna Lauesen 66 Chrfld. Gend.

* Maria Juhl 65 Tyrstrup Skom.

* Mgr. Kjær 64 Aller

1875

Christopha Troye 63 Bergen

Elvine M. Olsen 63 Trondh. Skibsf.

Henriette Smith 62 Arendal Reder

Marie C. Petersen 61 Bylderup Gdm.

Amalie Helbling 61 Surrey

* Marie W. Posselt 66 S-skovgd. Gdm.

* Ellen Dahl 61 Tyrstrupgd.

* Gyda Ravn 66 Tyrstrup Dyrl.

* Charl. Bock 67 Chrfld. Gdm.

* Elvine Schau 68 Chrfld. Gdm.

* Therese Barsge 66 Skovbøll. (Gdm.

\section{6}

Mor. Birck

Anna Loversen

Julie A. Smith

Chrestiane Smith

Inanda Dahl

.Jesm.e Sørensen

63 Arendal

6.) Arendal

64 Arendal

61 Porsgrund

65 Moss

68 Drenderup Iddm.

Marie Andersen

61 Mølmark Husm.

Emmy Ick

63 Häverwisch

Jane Stahlschmidt 60 London

Flor. Stahlschmidt 61 London

* Cathr. Schmidt 62 Frørup

* Dor.a S. Schmidt 66 Frørup

* Emilie Schmidt 68 Frørup

Mægl.

Mægl.

(idm.

Gdm.

Gdm.

* Soph. Buchholdt 66 Fauervraa Kbm.?

* Marie Jørgensen 63 Brændur Gdm.

* Karen Lund 67 Chrfld. Toldk.

* Aug.Schwarzrock69 Chrfld. Gend.

* Flor.e Schlünsen 65 Chrfld. Postk.?

* Charl. Bock 67 Bobøl Gdm.

\section{7}

DagnyGronneberg 67 Kbhn.

Thora Petersen 62 Staulo
Ior.a Bruun 63 Marstrup (idm.

Anne M. Winther (i4 Ballum Gdm.

Aug.e Matzen $\quad 64$

Ina Jones 63 England

* Cathr. Juhl 64 Tyrstrup Lage

* Ellen Dall 61 Tyrstrup Gdm.

* Cathr. Erichsen 70 Chrfld. Drejer

* Marie Schütt 65 Vonsild Gdm.

* Mgd. Sarensen 64 Seggel. (idm.

* Chr.e F.Schmidt 63 Frørup (idm.

* Fre.e Hansen 60 Hadersl. Læge

\section{8}

Henr. H. Schmidt 6:3 Arendal Bogtr.

Cecilie Jacobsen (64 Hadersl. Orgelb.

Marie Marcussen 63 Aabenraa Orgelb.

* Carol. Jørgensen 68 Stenderup Gdm.

* Carol. Damgaard 67 Tapsur Gdm.

* Marie Damgaard 68 Tapsur Gdin.

* Dor.a Danielsen 62 Sneumgd. Propr.

* Anna Hagge $\quad 66$

* Soph. Hagge 70

* Emma Johansen 72

\section{9}

Carol. Stahnke 69 Kolding Kbm. Hanna Bollemose 68 Oslo Kbm.

- Meta Barsøe 70 Skovholl Gdm.

- Elise Barsøe 71 Skovbøll (Ydm.

* Martha Leschke 68

\section{0}

Laura Jensen 67 Kolding Kbm.

Agnes Samson $\quad 64$

Mary Scott 64 Bristol

Mgr. Mc. Callum 64 London

* Henr.e C. Bæk 64 Aller

- Elisab. Ellegaard 66 Anslet Klm.

- Anita Barkog 68

* Alfred.e Møller 67 Faustrup Kbm.

* FriedaA.Schmidt 72 Frørup Sgnf.

* Math. Danielsen 64 Vojensgd. Forp.

1881

Atlantra Hentzen 69 Kolding

(xeorgia Lorenzen 68 ('hristinedalPropr.

* Helene Andresen 67 
* Marie Tippmann 65 Trondh. Soc.A.

\section{2}

Frieda Schmertz 69

* Gertr. Blume 74 Chrfld.

* Anna Becker 69 Aller Præst

* Maria Becker 71 Aller Præst

1883

* Charl. Becker 72 Aller Præst

* Amalie Becker 73 Aller Præst

* Elly Hoffmann 75 Tyrstrup- Bryggaard gem.

* Amanda Sievers 74 Hakkelb. (Yend.

* Chr.e Erichsen 75 Chrfld. Drejer

* Math.e Danielsen 67 Augusten- Forp. hof

\section{4}

Joh. E. Petersen

* Anna Juhl

* Anna Nissen

* Gertr. Padel

\section{5}

Aug.e Russ

Mary J. Juhler

Elwine Juhler

Cæcilie Kreich

69 Stepping Møll.

74 Brabæk Gdm.

72 Bøgeskov Gdm.

76 S. Afrika Br.M.

Else Schnarcke

* Anna Møller

71 Oslo

71 Løjt

73 Løjt

75 Kiel

75 Danzig

73 Errested Gdm.

* Marie Refshauge 73 Hvinderup Gdm

* Anna Hansen 77 Skovrup Gdm.

\section{6}

Linnhe Janssen

73 Hadersl. Læge

Marie K. Drews

72 Wessel. Lærer buren

* Meta Beck

* Bertha Kloss

78 Tyrstrup Bager

76 Tyrstrup Gend.

* Lieschen Kloss 76 Tyrstrup Gend.

* Cam. Schmidt 78 Frørup Sgnf.

*Clara E.Wilckens 77 Kabdrup Gdm.

\section{7}

Wera Nürnberg') 72 Kbhn.

Petra Holm 72 Oslo Postk.
Andrea Arentz 70 Trondh.

Ella Stange $\quad 75$ Flensb.

* Anna Sohl 75 Tagkær Ldm.

* Maria Hamann 74 Brabæk Forp.

* Anna Vülkel 79 Hokkelb. Toldk.

* Mgr. Møller 79 Faustrup Kbm.

* Anna Kylling 72 Bøgeskov Gdm.

* Emilie Freitag 74 Chrfld. Toldk.

* Jenny Holm 79 Chrfld. Skbf.

* Hanna Erichsen 79 Chrfld. Drejer

* Irmg. Padel 78 S. Afrika Br.M.

2) Jedinde, debt i Chrfld.

1888

Emma Lorenzen $75 \mathrm{Chr}$-inedal Propr.

Maria Arentz 72 Trondh.

Soph. Castberg 75 Trondh.

Märtha Ryding 76 Hernösand

Carol. Lausen 74 Hadersl. Gæstg.

Mgr. Jensen 73 Sønderb. Guld.

Soph. Biehl 74 Sønderb. Skbf.

*Ing. Schmidt 74 Sønderballe Gdm.

* Cathr. Ravn 74 Ringtved Gdm.

- Freya Jessen 76 Brændur Gdm.

* Hedw. Dinse 80 Tyrstrup Gend.

* Maria König $\mathbf{7 4}$ Høkkelb. Toldk. (f. i Broager)

* Anna Dinsen 75 Tagkær Skræ.

* Gustava Petersen 76 Stepping Møll.

\section{9}

Elisab.Rasmussen 74 Chr-sand

Camil. Rasmussen $76 \mathrm{Chr}$-sand

Louise Lohrbauer 74 Oslo

* Chr.e Jessen 76 Bylderup Gdm.

* Else Padel York Br.M.

\section{0}

Dora Boysen $\quad 76$ Altona

* Charl. Paulsen 76 Taps Læge

* Astrid Paulsen 80 Taps Læge

* Gertr. Claussen 82 Tyrstrup Præst

* Cæcilie Hansen 77 Højrup Gdm.

* Fre.e Erichsen 82 Chrfld, Drejer 


\section{Nogle statistiske Tal:}

Fortegnelsen omfatter ialt: 2457 Elever (1416 Drenge +1041 Piger),

deraf fra Danmark......... $545(254+291)$

Hertugdømmerne .......... $1035(618+417)$

Norge................ $376(163+213)$

Sverige............... $319(254+65)$

Tyskland ............... $33(18+16)$

England $\ldots \ldots \ldots \ldots \ldots \ldots \ldots \ldots .64(50+14)$

Andre Lande ............. $85 \quad 159+26)$

Hertil kommer fra Christiansfeld Menigheden: $465(243+222)$, som ikke er medtagne i denne Fortegnelse.

\section{Forkortelser:}

(af tekniske Grunde ikke helt ensartede)

Adj. Adjunkt

Adm. Admiral

Amtm. Amtimanid

Amtisf. Amtsforvalter

Apot. Apoteker

Arbm. Arbejdsimand

Audit. Auditør

Aas. Assessor

Bnikım. Bankmand

Bgtr. Bogtrykker

Brand. Branddirektør

Br. A. Brødre Arbejder

Brgm. Bongmester

Br. M. Brødne Missionær

Br. Pr. Brordre Præst

Bruk. Brulksplatron

Brukf. Brukisforvalter

Brygg. Brygger

Byf. Byfoged

Bygm. Bygmester

Dia-A. Diaspora Arbejider

Dir. Direktør

Domm. Dommer
Et. R. Etats Raad

Fabr. Fabrikant

Farv. Farver

Fonp. Forptagter

Forv. Forvalter

Fotogr. Fotograf

Frih. Friherre

Garv. Garver

Gdm. Gaandmand

Gend. Gendarm

Gen. Lt. General Lojtnant

Glrm. Gilarmester

Gods. Godsejer

Gross. Grosiserer

Grtn. Gartner

Gstg. Grestgiver

Guld. Guldsmed

Hatt. Hattemager

Hesteh. Hestehandler

Hoiskr. Hoisekræmmier

Hrrf. Herredsfoged

Husf. Husfoged

Husm. Husmand 
Insp. Inspektør

Instrm. Instrumentmager

Jern. Jernværksejer

Ju. R. Justits Raad

Kam. R. Kammer Raad

Kanc. R. Kancelli Raad

Kass. Kasserer

Kibm. Kobmand

Kimd. Kommandør

Kmrj. Kammerjun'ker

Knipl. Kniplingshandler

Knsil. Konsul

Kntr. Kontrollør

Kolkt. Kollektør

Kond. Konditor

Kpt. Kaptajn

Kptlt. Kaptajnløjtnant

Krigs R. Krigs Raad

Kæm. Kæmner

Lrdm. Landmand

Lensf. Lensfoged

Lnıdf. Landfonged

Lt. Løjtnant

Mejer. Mejerist

Mægl. Mægler

Møll. Møller

Obst. Oberst

Orgib. Orgelbygger

Port. Portier

Postk. Postkontrollør

Postm. Postmester

Prlt. Premierløjtnant

Prof. Professor

Prok. Prokurist
Propr. Proprietær

Prov. Provst

Raadm. Raadmand

Rebsl. Rebslager

Ritm. Ritmester

Sekr. Sekretær

Sgnf. Sognefoged

Skbb. Skibsbygger

Skbf. Skibsffrrer

Skom. Skomager

Skræ. Skræder

Sksp. Skuespiller

Slagt. Slagter

Snedk. Snedker

Soc. A. Societets Arbejrder

Soc. F. Societets Forstander

S. Skr. Soren Skriver

Styrm. Styrmand

Sadel. Sadelimager

Sagf. Sagfører

Slotsf. Slotsforvalter

St. R. Strats Raad

Tandl. Tandlæge

Tegl. Teglværksejer

Tldasis. Toldassistent

Tldif. Tolddorvalter

TIdk. Tioldkontrollør

Tngiskr. Tingskriver

Tømr. Tømrer

U. off. Underofficer

$\mathrm{L}^{\top} \mathrm{l} \cdot \mathrm{m}$. Urmager

Vinh. Vinhandler

Vognm. Vognmand

Værkm. Værkmester 